Kragujevac Journal of Mathematics

Volume 39(1) (2015), Pages 53-71.

\title{
INTEGRAL EQUATIONS METHOD AND THE TRANSMISSION PROBLEM FOR THE STOKES SYSTEM
}

\author{
D. MEDKOVÁ ${ }^{1}$
}

\begin{abstract}
The transmission problem for the Stokes system is studied: $\Delta \mathbf{v}_{ \pm}=$ $\nabla p_{ \pm}, \nabla \cdot \mathbf{v}_{ \pm}=0$ in $G_{ \pm}, \mathbf{v}_{+}-\mathbf{v}_{-}=\mathbf{g}, a_{+} T\left(\mathbf{v}_{+}, p_{+}\right) \mathbf{n}-a_{-} T\left(\mathbf{v}_{-}, p_{-}\right) \mathbf{n}=\mathbf{f}$ on $\partial G_{+}$. Here $G_{+} \subset R^{3}$ is a bounded open set with Lipschitz boundary and $G_{-}$is the corresponding complementary open set. Using the integral equation method we study the problem in homogeneous Sobolev spaces. Under assumption that $\partial G_{+}$ is of class $\mathcal{C}^{1}$ we study this problem also in Besov spaces and $L^{q}$-solutions of the problem. We show the unique solvability of the problem. Moreover, we solve the corresponding boundary integral equations by the successive approximation method.
\end{abstract}

\section{INTRODUCTION}

The transmission problem is a very important problem and there are lots of papers devoted to this topic (see for example [18], [39], [2], [7], [38], [10]). This problem occurs in the case of contact of two media with different material constants. Very fruitful is to study this problem using the integral equation method (see [4], [35], [12], [11], [13], [34], [5]). Many papers study the Brinkman transmission problem and the Stokes-Brinkman transmission problem by the integral equation method ([21], [22], [23], [25], [20], [24]). The transmission problem for the Stokes system was studied by the integral equation method in [3], [36]. The following transmission problem was studied:

$$
\begin{gathered}
\Delta \mathbf{v}_{ \pm}=\nabla p_{ \pm} \quad \text { in } \quad G_{ \pm}, \quad \nabla \cdot \mathbf{v}_{ \pm}=0 \quad \text { in } \quad G_{ \pm} \\
\mathbf{v}_{+}-\mathbf{v}_{-}=\mathbf{g}, \quad a_{+} T\left(\mathbf{v}_{+}, p_{+}\right) \mathbf{n}-a_{-} T\left(\mathbf{v}_{-}, p_{-}\right) \mathbf{n}=\mathbf{f} \quad \text { on } \partial G . \\
\mathbf{v}_{-}(\mathbf{x})=O\left(|\mathbf{x}|^{-1}\right), \quad \nabla \mathbf{v}_{-}(\mathbf{x})=O\left(|\mathbf{x}|^{-2}\right), p_{-}(\mathbf{x})=O\left(|\mathbf{x}|^{-2}\right) \quad \text { as }|\mathbf{x}| \rightarrow \infty .
\end{gathered}
$$

Key words and phrases. Transmission problem, Stokes system, integral equation method. 2010 Mathematics Subject Classification. Primary: 35Q35. Secondary: 65N38

Received: Decembar 5, 2013

Accepted: April 4, 2015. 
Here $G=G_{+} \subset R^{3}$ is a bounded open set with Lipschitz boundary $\partial G, G_{-}:=$ $R^{3} \backslash \mathrm{cl} G_{+}$its complement with $\partial G_{-}=\partial G$, where $\mathrm{cl} G_{+}$denotes the closure of $G_{+}$ and $\partial G$ the boundary of $G, \mathbf{n}=\mathbf{n}^{G}$ is the outward unit normal of $G_{+}, a_{+}, a_{-}$are positive constants, and

$$
T(\mathbf{v}, p)=2 \hat{\nabla} \mathbf{v}-p I
$$

is the stress tensor. Here $I$ denotes the identity matrix and

$$
\hat{\nabla} \mathbf{v}=\frac{1}{2}\left[\nabla \mathbf{v}+(\nabla \mathbf{v})^{T}\right]
$$

is the strain tensor, with $(\nabla \mathbf{v})^{T}$ as the matrix transposed to $\nabla \mathbf{v}=\left(\partial_{j} v_{k}\right),(k, j=$ $1,2,3)$, and $\nabla \cdot \mathbf{v}=\partial_{1} v_{1}+\partial_{2} v_{2}+\partial_{3} v_{3}$ denotes the divergence of $\mathbf{v}$. For $1<q \leq 2$ and $\mathbf{f} \in$ $L^{q}\left(\partial \Omega, R^{3}\right)$ and $\mathbf{g} \in W^{1, q}\left(\partial \Omega, R^{3}\right)$ it was shown that there exists a unique $L^{q}$-solution of the transmission problem (1.1), (1.2), (1.3). It means that the nontangential maximal functions of $\mathbf{v}_{ \pm}, \nabla \mathbf{v}_{ \pm}$and $p_{ \pm}$are in $L^{q}(\partial \Omega)$ and the transmission conditions (1.2) are satisfied in the sense of the nontangential limit. (See [36], Theorem 9.2.1.)

The integral equation method is a basis of calculation of a solution of the problem by the boundary element method (see [14], [17], [43], [40]). The famous article [44] studies the Neumann problem for elliptic systems by the integral equation method. O. Steinbach and W. L. Wendland looked for a solution in the form of a single layer potential. They proved that the corresponding integral equation can be solved by the successive approximation. The goal of this paper is to prove a similar result for the transmission problem for the Stokes system.

First we study a solution $\mathbf{v}_{ \pm} \in \tilde{W}^{1,2}\left(G_{ \pm}, R^{3}\right), p_{ \pm} \in L^{2}\left(G_{ \pm}\right)$of the problem (1.1), (1.2) for $G_{+}$with Lipschitz boundary. Here the homogeneous Sobolev space $\tilde{W}^{1,2}\left(G_{ \pm}\right)$is defined by $\tilde{W}^{1,2}\left(G_{ \pm}\right)=\left\{u \in L^{6}\left(G_{ \pm}\right) ; \partial_{j} u \in L^{2}\left(G_{ \pm}\right)\right\}$. (It is well known that the study of the problem in the standard Sobolev space $W^{1,2}\left(G_{ \pm}\right)=$ $\left\{u \in L^{2}\left(G_{ \pm}\right) ; \partial_{j} u \in L^{2}\left(G_{ \pm}\right)\right\}$failes. Remark that $W^{1,2}\left(G_{ \pm}\right)$is a dense subspace of $\tilde{W}^{1,2}\left(G_{ \pm}\right)$, and $\tilde{W}^{1,2}\left(G_{+}\right)=W^{1,2}\left(G_{+}\right), \tilde{W}^{1,2}\left(G_{-}\right) \neq W^{1,2}\left(G_{-}\right)$.) We show that the transmission problem is uniquely solvable and

$$
\mathbf{v}_{ \pm}=D_{G} \mathbf{g}+E_{G} \mathbf{\Psi}, \quad p_{ \pm}=\Pi_{G} \mathbf{g}+Q_{G} \boldsymbol{\Psi}
$$

with

$$
\boldsymbol{\Psi}=T\left(\mathbf{v}_{+}, p_{+}\right) \mathbf{n}-T\left(\mathbf{v}_{-}, p_{-}\right) \mathbf{n} .
$$

Here $\left(D_{G} \mathbf{g}, \Pi_{G} \mathbf{g}\right)$ is the hydrodynamical double layer potential with density $\mathbf{g}$ and $\left(E_{G} \boldsymbol{\Psi}, Q_{G} \boldsymbol{\Psi}\right)$ is the hydrodynamical single layer potential with the density $\boldsymbol{\Psi}$. So, it is enough to calculate $\boldsymbol{\Psi}$. The density $\boldsymbol{\Psi}$ satisfies the integral equation

$$
\boldsymbol{\Psi}=\frac{2\left(a_{+}-a_{-}\right)}{\left(a_{+}+a_{-}\right)} K_{G}^{\prime} \boldsymbol{\Psi}+\frac{2}{a_{+}+a_{-}} \mathbf{F}
$$


(for the definition of the operator $K_{G}^{\prime}$ see $\S 3$; for the definition of $\mathbf{F}$ see $\S 5$ ). We show that this equation is uniquely solvable. If $\boldsymbol{\Psi}_{0} \in H^{-1 / 2}\left(\partial G, R^{3}\right)$ is fixed

$$
\boldsymbol{\Psi}_{k}=\frac{2\left(a_{+}-a_{-}\right)}{\left(a_{+}+a_{-}\right)} K_{G}^{\prime} \boldsymbol{\Psi}_{k-1}+\frac{2}{a_{+}+a_{-}} \mathbf{F}
$$

then $\Psi_{k} \rightarrow \Psi$ and

$$
\left\|\Psi_{k}-\boldsymbol{\Psi}\right\|_{H^{-1 / 2}\left(\partial G, R^{3}\right)} \leq C \alpha^{k}\left(\left\|\Psi_{0}\right\|_{H^{-1 / 2}\left(\partial G, R^{3}\right)}+\|F\|_{H^{-1 / 2}\left(\partial G, R^{3}\right)}\right),
$$

where

$$
\alpha=\frac{\max \left(a_{+}, a_{-}\right)}{a_{+}+a_{-}}<1
$$

and a constant $C$ depends only on $G, a_{+}$and $a_{-}$.

Under the assumption that $\partial G$ is of class $\mathcal{C}^{1}$ and $1<q<\infty$ we study an $L^{q}$-solution of the transmission problem (1.1), (1.2) accomplished with the condition

$$
\mathbf{v}_{-}(\mathbf{x}) \rightarrow 0, \quad p_{-}(\mathbf{x}) \rightarrow 0 \quad \text { as }|\mathbf{x}| \rightarrow \infty
$$

instead of the rather artificial condition (1.3). We show the unique solvability of the problem and the representation (1.4), (1.5). The density $\boldsymbol{\Psi} \in L^{q}\left(\partial G, R^{3}\right)$ is a unique solution of the equation (1.6). If $\boldsymbol{\Psi}_{0} \in L^{q}\left(\partial G, R^{3}\right)$ is fixed and $\boldsymbol{\Psi}_{k}$ is given by (1.7), then $\boldsymbol{\Psi}_{k} \rightarrow \boldsymbol{\Psi}$ and

$$
\left\|\Psi_{k}-\boldsymbol{\Psi}\right\|_{L^{q}\left(\partial G, R^{3}\right)} \leq C \alpha^{k}\left(\left\|\Psi_{0}\right\|_{L^{q}\left(\partial G, R^{3}\right)}+\|F\|_{L^{q}\left(\partial G, R^{3}\right)}\right)
$$

where $\alpha$ is given by (1.8) and a constant $C$ depends only on $G, a_{+}, a_{-}$and $q$.

Under the assumption that $\partial G$ is of class $\mathrm{C}^{1}$ and $0<s<1,1<q, t<\infty$ we study a solution of the transmission problem (1.1), (1.2), (1.9) in Besov space, i.e. for $\mathbf{f} \in$ $B_{s-1}^{q, t}\left(\partial G, R^{3}\right), \mathbf{g} \in B_{s}^{q, t}\left(\partial G, R^{3}\right)$ we look for $\mathbf{v}_{+} \in B_{s+1 / q}^{q, t}\left(G_{+}, R^{3}\right), p_{+} \in B_{s+1 / q-1}^{q, t}\left(G_{+}\right)$, $\mathbf{v}_{-} \in B_{s+1 / q}^{q, t}\left(G_{-} \cap B(0 ; r), R^{3}\right), p_{-} \in B_{s+1 / q-1}^{q, t}\left(G_{-} \cap B(0 ; r)\right)$ for all $r>0$. (Here $B(\mathbf{x} ; r)=\left\{\mathbf{y} \in R^{3} ;|\mathbf{x}-\mathbf{y}|<r\right\}$.) We show the unique solvability of the problem and the representation (1.4), (1.5). The density $\Psi \in B_{s-1}^{q, t}\left(\partial G, R^{3}\right)$ is a unique solution of the equation (1.6). If $\boldsymbol{\Psi}_{0} \in B_{s-1}^{q, t}\left(\partial G, R^{3}\right)$ is fixed and $\boldsymbol{\Psi}_{k}$ is given by (1.7), then $\Psi_{k} \rightarrow \Psi$ and

$$
\left\|\Psi_{k}-\Psi\right\|_{B_{s-1}^{q, t}\left(\partial G, R^{3}\right)} \leq C \alpha^{k}\left(\left\|\Psi_{0}\right\|_{B_{s-1}^{q, t}\left(\partial G, R^{3}\right)}+\|F\|_{B_{s-1}^{q, t}\left(\partial G, R^{3}\right)}\right),
$$

where $\alpha$ is given by (1.8) and a constant $C$ depends only on $G, a_{+}, a_{-}, s, q, t$.

Under the assumption that $\partial G$ is of class $\mathcal{C}^{1}$ and $1<q<\infty$ we study a solution of the transmission problem (1.1), (1.2), (1.9) such that $\mathbf{v}_{ \pm} \in D^{1, q}\left(G_{ \pm}\right), p \in L^{q}\left(G_{ \pm}\right)$, where the homogeneous Sobolev space is defined by $D^{1, q}\left(G_{ \pm}\right)=\left\{u ; \partial_{j} u \in L^{q}\left(G_{ \pm}\right)\right\}$. First, we show that such solution is a solution in a Besov space, i.e. $\mathbf{v}_{+} \in B_{1}^{q, q}\left(G_{+}, R^{3}\right)$, $p_{+} \in B_{0}^{q, q}\left(G_{+}\right), \mathbf{v}_{-} \in B_{1}^{q, q}\left(G_{-} \cap B(0 ; r), R^{3}\right), p_{-} \in B_{0}^{q, q}\left(G_{-} \cap B(0 ; r)\right)$ for all $r>0$. So, we have the representation (1.4), (1.5). The density $\Psi \in W^{-1 / q, q}\left(\partial G, R^{3}\right)=$ 
$B_{-1 / q}^{q, q}\left(\partial G, R^{3}\right)$ is a unique solution of the equation (1.6). If $\boldsymbol{\Psi}_{0} \in W^{-1 / q, q}\left(\partial G, R^{3}\right)$ is fixed and $\boldsymbol{\Psi}_{k}$ is given by (1.7), then $\boldsymbol{\Psi}_{k} \rightarrow \boldsymbol{\Psi}$ and

$$
\left\|\Psi_{k}-\Psi\right\|_{W^{-1 / q, q}\left(\partial G, R^{3}\right)} \leq C \alpha^{k}\left(\left\|\Psi_{0}\right\|_{W^{-1 / q, q}\left(\partial G, R^{3}\right)}+\|F\|_{W^{-1 / q, q}\left(\partial G, R^{3}\right)}\right),
$$

where $\alpha$ is given by (1.8) and a constant $C$ depends only on $G, a_{+}, a_{-}$and $q$. A more complicated problem is the solvability of the problem. If $3 / 2<q<\infty$ then the problem (1.1), (1.2), (1.9) is uniquely solvable. If $1<q \leq 3 / 2$ then the problem $(1.1),(1.2),(1.9)$ is solvable if and only if $\langle\mathbf{f}, \mathbf{c}\rangle=0$ for each constant vector $\mathbf{c}$ and a solution is unique.

\section{Formulation of the problem}

We shall study the transmission problem (1.1), (1.2). Instead of the artificial condition (1.3) we use a more natural condition.

First we define a scale of strong solutions (i.e. $L^{q}$-solutions). Let $\Omega$ be an open set with Lipschitz boundary. If $\mathbf{x} \in \partial \Omega, a>0$ denote the non-tangential approach regions of opening $a$ at the point $\mathbf{x}$ by

$$
\Gamma_{a}^{\Omega}(\mathbf{x}):=\{\mathbf{y} \in \Omega ;|\mathbf{x}-\mathbf{y}|<(1+a) \operatorname{dist}(\mathbf{y}, \partial \Omega)\} .
$$

If now $\mathbf{v}$ is a vector function defined in $\Omega$ we denote the non-tangential maximal function of $\mathbf{v}$ on $\partial \Omega$ by

$$
\mathbf{v}_{\Omega}^{*}(\mathbf{x}):=\sup \left\{|\mathbf{v}(\mathbf{y})| ; \mathbf{y} \in \Gamma_{a}^{\Omega}(\mathbf{x})\right\} .
$$

If $\mathbf{x} \in \partial \Omega, \Gamma(\mathbf{x})=\Gamma_{a}^{\Omega}(\mathbf{x})$ then

$$
\mathbf{v}(\mathbf{x})=\lim _{\substack{\mathbf{y} \rightarrow \mathbf{x} \\ \mathbf{y} \in \Gamma(\mathbf{x})}} \mathbf{v}(\mathbf{y})
$$

is the non-tangential limit of $\mathbf{v}$ with respect to $\Omega$ at $\mathbf{x}$.

Let now $1<q<\infty, \mathbf{f} \in L^{q}\left(\partial G, R^{3}\right)$, $\mathbf{g} \in W^{1, q}\left(\partial G, R^{3}\right)$. We say that $\mathbf{v}_{ \pm}, p_{ \pm}$ defined on $G_{ \pm}$is an $L^{q}$-solution of the transmission problem (1.1), (1.2), (1.9) if $\mathbf{v}_{+}, p_{+}$satisfy (1.1) and (1.9); $\left(\nabla \mathbf{v}_{ \pm}\right)^{*}, p_{ \pm}^{*}, \mathbf{v}_{ \pm}^{*}$ are from $L^{q}\left(\partial G, R^{1}\right)$; for almost all $\mathbf{x} \in \partial G$ there exist the non-tangential limits of $\mathbf{v}_{ \pm}, \nabla \mathbf{v}_{ \pm}$and $p_{ \pm}$at $\mathbf{x}$ and the condition (1.2) is fulfilled in the sense of the nontangential limit a.e. on $\partial G$.

Now we formulate a weak solution $\mathbf{v}_{ \pm} \in \tilde{W}^{1,2}\left(G_{ \pm}, R^{3}\right), p_{ \pm} \in L^{2}\left(G_{ \pm}\right)$in the homogeneous Sobolev space for $\mathbf{f} \in H^{-1 / 2}\left(\partial G, R^{3}\right), \mathbf{g} \in H^{1 / 2}\left(\partial G, R^{3}\right)$.

Denote by $D^{1,2}\left(R^{3}\right)$ the space of all functions $u \in L_{\text {loc }}^{2}\left(R^{3}\right)$ such that $\partial_{j} u \in L^{2}\left(R^{3}\right)$ in the sense of distributions for each $j=1,2,3$. Then $D^{1,2}\left(R^{3}\right)$ is a Banach space with the norm

$$
\|u\|_{L^{1,2}\left(R^{3}\right)}=\sqrt{\int_{G}|u|^{2} d \mathcal{H}_{m}+\int_{R^{3}}|\nabla u|^{2} d \mathcal{H}_{m}}
$$


(see [30], §1.5.3). Denote by $\mathcal{C}_{c}^{\infty}\left(R^{3}\right)$ the space of all infinitely differentiable functions in $R^{3}$ with compact support. Denote by $\tilde{W}^{1,2}\left(R^{3}\right)$ the closure of $\mathcal{C}_{c}^{\infty}\left(R^{3}\right)$ in $D^{1,2}\left(R^{3}\right)$. The space $D^{1,2}\left(R^{3}\right)$ is the direct sum of $\tilde{W}^{1,2}\left(R^{3}\right)$ and the space of constant functions (see [8], p. 155). If we put

$$
\|u\|_{\tilde{W}^{1,2}\left(R^{3}\right)}=\|\nabla u\|_{L^{2}\left(R^{3}\right)},
$$

then this norm is equivalent with the norm induced from $D^{1,2}\left(R^{3}\right)$ (see [30], §1.5.2 and [30], §1.5.3). According to [26], Lemma 2.2 we have $\tilde{W}^{1,2}\left(R^{3}\right)=\left\{u \in L^{6}\left(R^{3}\right) ; \nabla u \in\right.$ $\left.L^{2}\left(R^{3} ; R^{3}\right)\right\}$. For an open set $\Omega$ denote by $\tilde{W}^{1,2}(\Omega)$ the space of restrictions of functions from $\tilde{W}^{1,2}\left(R^{3}\right)$ onto $\Omega$. Denote

$$
\|u\|_{\tilde{W}^{1,2}(\Omega)}=\inf \left\{\|v\|_{\tilde{W}^{1,2}\left(R^{3}\right)} ; v=u \text { on } \Omega\right\} .
$$

Then $\tilde{W}^{1,2}(\Omega)$ is a Banach space. If $\Omega$ is a bounded open set with Lipschitz boundary then $\tilde{W}^{1,2}(\Omega)=H^{1}(\Omega)$ and both norms are equivalent. If $\Omega$ is an unbounded domain with compact Lipschitz boundary then $\|\nabla u\|_{L^{2}(\Omega)}$ is an equivalent norm in $\tilde{W}^{1,2}(\Omega)$. According to [6], Chapter XI, Part B, $\S 1$ we have $\tilde{W}^{1,2}(\Omega)=\left\{u ; \partial_{j} u \in L^{2}(\Omega), u(x)(1+\right.$ $\left.\left.|\mathbf{x}|^{2}\right)^{-1 / 2} \in L^{2}(\Omega)\right\}$.

Using weak characterizations of the Neumann boundary condition for the Stokes system in $G_{+}$and in $G_{-}$(see [32]) and the fact that $\mathbf{n}^{G_{-}}=-\mathbf{n}^{G_{+}}$we give a weak formulation of the transmission problem for the Stokes system (1.1), (1.2):

Let $\mathbf{g} \in H^{1 / 2}\left(\partial G, R^{3}\right), \mathbf{f} \in H^{-1 / 2}\left(\partial G, R^{3}\right)$. We say that $\mathbf{v}_{ \pm} \in \tilde{W}^{1,2}\left(G_{ \pm}, R^{3}\right)$, $p_{ \pm} \in L^{2}\left(G_{ \pm}, R^{1}\right)$ is a weak solution of the transmission problem for the Stokes system (1.1), (1.2) if $\nabla \cdot \mathbf{v}_{ \pm}=0, \mathbf{v}_{+}-\mathbf{v}_{-}=\mathbf{g}$ on $\partial G$ in the sense of traces and

$a_{+} \int_{G_{+}}\left(2 \hat{\nabla} \mathbf{v}_{+} \cdot \hat{\nabla} \mathbf{w}-p_{+}(\nabla \cdot \mathbf{w})\right) d \mathcal{H}_{3}+a_{-} \int_{G_{-}}\left(2 \hat{\nabla} \mathbf{v}_{-} \cdot \hat{\nabla} \mathbf{w}-p_{-}(\nabla \cdot \mathbf{w})\right) d \mathcal{H}_{3}=\langle\mathbf{f}, \mathbf{w}\rangle$

for all $\mathbf{w} \in \tilde{W}^{1,2}\left(R^{3}, R^{3}\right)$.

\section{The SURFACE POTENTIALS}

The aim of this section is to assemble some basic facts on hydrodynamical potentials. For $\mathbf{x} \in R^{3}$, and $j, k=1,2,3$ define

$$
E_{j k}(\mathbf{x})=\frac{1}{8 \pi}\left[\frac{\delta_{j k}}{|\mathbf{x}|}+\frac{x_{j} x_{k}}{|\mathbf{x}|^{3}}\right], \quad Q_{k}(\mathbf{x})=\frac{x_{k}}{4 \pi|\mathbf{x}|^{3}} .
$$

If $\boldsymbol{\Psi}$ is a vector function (or distribution) supported on $\partial G$ define the hydrodynamical single layer potential with density $\Psi$ by

$$
\left(E_{G} \Psi\right)(\mathbf{x})=\langle\Psi, E(\mathbf{x}-\cdot)\rangle=\int_{\partial G} E(\mathbf{x}-\mathbf{y}) \Psi(\mathbf{y}) \mathrm{d} \mathcal{H}_{2}(\mathbf{y})
$$


and the corresponding pressure by

$$
\left(Q_{G} \boldsymbol{\Psi}\right)(\mathbf{x})=\langle\Psi, Q(\mathbf{x}-\cdot)\rangle=\int_{\partial G} Q(\mathbf{x}-\mathbf{y}) \Psi(\mathbf{y}) \mathrm{d} \mathcal{H}_{2}(\mathbf{y}) .
$$

Then $E_{G} \boldsymbol{\Psi} \in C^{\infty}\left(R^{3} \backslash \partial G, R^{3}\right), Q_{G} \boldsymbol{\Psi} \in C^{\infty}\left(R^{3} \backslash \partial G, R^{1}\right), \nabla Q_{G} \boldsymbol{\Psi}-\Delta E_{G} \boldsymbol{\Psi}=0$, $\nabla \cdot E_{G} \Psi=0$ in $R^{3} \backslash \partial G$. We have the following decay behavior as $|\mathbf{x}| \rightarrow \infty$ :

$$
\begin{gathered}
E_{G} \boldsymbol{\Psi}(\mathbf{x})=O\left(|\mathbf{x}|^{-1}\right), \\
Q_{G} \boldsymbol{\Psi}(\mathbf{x}),\left|\left(\nabla E_{G} \boldsymbol{\Psi}\right)(\mathbf{x})\right|=O\left(|\mathbf{x}|^{-2}\right) .
\end{gathered}
$$

If $\boldsymbol{\Psi} \in L^{q}\left(\partial G, R^{3}\right), 1<q<\infty$ then $E_{G} \boldsymbol{\Psi}(\mathbf{x})$ makes sense for almost all $\mathbf{x} \in \partial G$ and $E_{G} \boldsymbol{\Psi}(\mathbf{x})$ is the non-tangential limit of $E_{G} \boldsymbol{\Psi}$ (see [29], Corollary 3.3). The nontangential maximal function of $E_{G} \boldsymbol{\Psi}, \nabla E_{G} \boldsymbol{\Psi}, Q_{G} \boldsymbol{\Psi}$ with respect to $G_{+}$and $G_{-}$is in $L^{q}(\partial G)$ (see [19], Lemma 2.1.4). Moreover, $E_{G}$ is a bounded linear operator from $L^{q}(\partial G)$ to $W^{1, q}(\partial G)$ (see [29], Corollary 3.3) and $E_{G} \boldsymbol{\Psi} \in W^{1, q}\left(G, R^{3}\right), Q_{G} \boldsymbol{\Psi} \in L^{q}(G)$ (see [29], Theorem 4.4).

If $0<s<1$ and $1<q, t<\infty$ then $E_{G}$ is a bounded linear operator from $B_{s-1}^{q, t}\left(\partial \Omega, R^{3}\right)$ to $B_{s+1 / q}^{q, t}(\Omega)$ and $Q_{G}$ is a bounded linear operator form $B_{s-1}^{q, t}\left(\partial \Omega, R^{3}\right)$ to $B_{s+1 / q-1}^{q, t}(\Omega)$ (see [29], Theorem 4.4). Moreover, $E_{G}$ is a bounded linear operator from $B_{s-1}^{q, t}\left(\partial \Omega, R^{3}\right)$ to $B_{s}^{q, t}(\partial \Omega)$ and $E_{G} \boldsymbol{\Psi}$ is the trace of $E_{G} \boldsymbol{\Psi}$ (see [29], Proposition 4.5). In particular, if $s \neq 1-1 / q$ then $E_{G}$ is a bounded linear operator from $W^{s-1, q}\left(\partial G, R^{3}\right)=$ $B_{s-1}^{q, q}\left(\partial G, R^{3}\right)$ to $W^{s+1 / q, q}\left(G, R^{3}\right)=B_{s+1 / q}^{q, q}\left(G, R^{3}\right)$; and $Q_{G}$ is a bounded linear operator from $W^{s-1, q}\left(\partial G, R^{3}\right)=B_{s-1}^{q, q}\left(\partial G, R^{3}\right)$ to $W^{s+1 / q-1, q}(G)=B_{s+1 / q-1}^{q, q}(G)$. By the interpolation argument we obtain this result also for $s=1-1 / q$ (see [1], p. 248 and [41], Chapter 5, Theorem 5). If $\boldsymbol{\Psi} \in H^{-1 / 2}\left(\partial G, R^{3}\right)=W^{-1 / 2,2}\left(\partial G, R^{3}\right)$ then the behavior at infinity gives $E_{G} \boldsymbol{\Psi} \in \tilde{W}^{1,2}\left(R^{3}, R^{3}\right), Q_{G} \boldsymbol{\Psi} \in L^{2}\left(R^{3}, R^{1}\right)$.

Remark that

$$
E_{G} \mathbf{n}^{G}=0 \text { in } R^{3}, \quad Q_{G} \mathbf{n}^{G}=-1 \text { in } G_{+}, \quad Q_{G} \mathbf{n}^{G}=0 \text { in } G_{-}
$$

(see for example [42], §3.2).

Now we define a hydrodynamical double layer potential. Fix $\mathbf{y} \in \partial G$ such that there is the unit outward normal $\mathbf{n}^{G}(\mathbf{y})$ of $G$ at $\mathbf{y}$. For $\mathbf{x} \in R^{3} \backslash\{\mathbf{y}\}, j, k \in\{1,2,3\}$ put

$$
\begin{aligned}
K_{j k}^{G}(\mathbf{x}, \mathbf{y}) & =\frac{3}{4 \pi} \frac{\left(y_{j}-x_{j}\right)\left(y_{k}-x_{k}\right)(\mathbf{y}-\mathbf{x}) \cdot \mathbf{n}^{G}(\mathbf{y})}{|\mathbf{x}-\mathbf{y}|^{5}} \\
\Pi_{k}^{G}(\mathbf{x}, \mathbf{y}) & =\frac{1}{2 \pi}\left\{-3 \frac{\left(y_{k}-x_{k}\right)(\mathbf{y}-\mathbf{x}) \cdot \mathbf{n}^{G}(\mathbf{y})}{|\mathbf{y}-\mathbf{x}|^{5}}+\frac{n_{k}^{G}(\mathbf{y})}{|\mathbf{y}-\mathbf{x}|^{3}}\right\} .
\end{aligned}
$$

For $\boldsymbol{\Psi}=\left[\Psi_{1}, \Psi_{2}, \Psi_{3}\right] \in L^{q}\left(\partial G, R^{3}\right)$ define the hydrodynamical double layer potential with density $\Psi$ by

$$
\left(D_{G} \boldsymbol{\Psi}\right)(\mathbf{x})=\int_{\partial G} K^{G}(\mathbf{x}, \mathbf{y}) \boldsymbol{\Psi}(\mathbf{y}) \mathrm{d} \mathcal{H}_{2}(\mathbf{y})
$$


and the corresponding pressure by

$$
\left(\Pi_{G} \boldsymbol{\Psi}\right)(\mathbf{x})=\int_{\partial G} \Pi^{G}(\mathbf{x}, \mathbf{y}) \Psi(\mathbf{y}) \mathrm{d} \mathcal{H}_{2}(\mathbf{y})
$$

in $R^{3} \backslash \partial G$. Then $D_{G} \boldsymbol{\Psi} \in C^{\infty}\left(R^{3} \backslash \partial G, R^{3}\right), \Pi_{G} \boldsymbol{\Psi} \in C^{\infty}\left(R^{3} \backslash \partial G, R^{1}\right)$ and $\nabla \Pi_{G} \boldsymbol{\Psi}-$ $\Delta D_{G} \boldsymbol{\Psi}=0, \nabla \cdot D_{G} \boldsymbol{\Psi}=0$ in $R^{3} \backslash \partial G$. We have the following decay behavior as $|\mathbf{x}| \rightarrow \infty$ :

$$
\begin{gathered}
\left(D_{G} \boldsymbol{\Psi}\right)(\mathbf{x})=O\left(|\mathbf{x}|^{-2}\right), \\
\left|\left(\nabla D_{G} \boldsymbol{\Psi}\right)(\mathbf{x})\right|, \quad \Pi_{G} \boldsymbol{\Psi}(\mathbf{x})=O\left(|\mathbf{x}|^{-3}\right) .
\end{gathered}
$$

Define

$$
K_{G} \boldsymbol{\Psi}(\mathbf{x})=\lim _{\epsilon \searrow 0} \int_{\partial G \backslash B(\mathbf{x} ; \epsilon)} K^{G}(\mathbf{x}, \mathbf{y}) \boldsymbol{\Psi}(\mathbf{y}) \mathrm{d} \mathcal{H}_{2}(\mathbf{y})
$$

on $\partial G$, where $B(\mathbf{x} ; \epsilon)=\{\mathbf{y} ;|\mathbf{x}-\mathbf{y}|<\epsilon\}$. Then $K_{G}$ is a bounded linear operator on $L^{q}\left(\partial G ; R^{3}\right)$ (see [29], Corollary 3.3). If $0<s<1$ and $1<q, t<\infty$ then $K_{G}$ is a bounded linear operator on $W^{1, q}(\partial G)$ (see [29], Proposition 3.5) and on $B_{s}^{q, t}(\partial G$ ) (see [29], Proposition 4.5). If $\boldsymbol{\Psi} \in L^{q}\left(\partial G ; R^{3}\right)$ then there exists the nontangential limit $\left[D_{G} \boldsymbol{\Psi}\right]_{+}(\mathbf{x})$ of $D_{G} \boldsymbol{\Psi}$ with respect to $G_{+}$and the nontangential limit $\left[D_{G} \boldsymbol{\Psi}\right]_{-}(\mathbf{x})$ of $D_{G} \boldsymbol{\Psi}$ with respect to $G_{-}$for almost all $\mathrm{x} \in \partial G$ and

$$
\left[D_{G} \boldsymbol{\Psi}\right]_{ \pm}(\mathbf{x})= \pm \frac{1}{2} \boldsymbol{\Psi}(\mathbf{z})+K_{G} \boldsymbol{\Psi}(\mathbf{z})
$$

(see [29], Proposition 3.2). Then $D_{G}: B_{s}^{q, t}(\partial G) \rightarrow B_{s+1 / q}^{q, t}(G)$ and the operator $\Pi_{G}: B_{s}^{q, t}(\partial G) \rightarrow B_{s+1 / q-1}^{q, t}(G)$ are bounded and $\left[D_{G} \boldsymbol{\Psi}\right]_{ \pm}$is the trace of $D_{G} \boldsymbol{\Psi}$ with respect to $G_{ \pm}$(see [29], Theorem 4.4). In particular, if $s \neq 1-1 / q$ then $D_{G}$ is a bounded linear operator from $W^{s, q}\left(\partial G, R^{3}\right)=B_{s}^{q, q}\left(\partial G, R^{3}\right)$ to $W^{s+1 / q, q}\left(G, R^{3}\right)=$ $B_{s+1 / q}^{q, q}\left(G, R^{3}\right) ; \Pi_{G}$ is a bounded linear operator from $W^{s, q}\left(\partial G, R^{3}\right)=B_{s}^{q, q}\left(\partial G, R^{3}\right)$ to $W^{s+1 / q-1, q}(G)=B_{s+1 / q-1}^{q, q}(G)$. By the interpolation argument we obtain this result also for $s=1-1 / q$ (see [1], p. 248 and [41], Chapter 5, Theorem 5). If $\boldsymbol{\Psi} \in H^{1 / 2}\left(\partial G, R^{3}\right)=W^{1 / 2,2}\left(\partial G, R^{3}\right)$ then the behavior at infinity gives $D_{G} \boldsymbol{\Psi} \in$ $\tilde{W}^{1,2}\left(G_{ \pm} ; R^{3}\right), \Pi_{G} \boldsymbol{\Psi} \in L^{2}\left(G_{ \pm}\right)$. If $\boldsymbol{\Psi} \in W^{1, q}\left(\partial G, R^{3}\right)$ then $\left(D_{\Omega} \boldsymbol{\Psi}\right)_{G}^{*}+\left(\nabla D_{\Omega} \boldsymbol{\Psi}\right)_{G}^{*}+$ $\left(\Pi_{G} \boldsymbol{\Psi}\right)_{G}^{*} \in L^{q}(\partial G)$ and there exist the non-tangential limits of $\nabla D_{\Omega} \boldsymbol{\Psi}$ and $\Pi_{G} \boldsymbol{\Psi}$ at almost all points of $\partial G$ (see [29], Proposition 3.4).

Denote by $K_{G}^{\prime}$ the adjoint operator of $K_{G}$. Then $K_{G}^{\prime}$ is a bounded linear operator on $L^{q}\left(\partial G, R^{3}\right)$ and on $B_{-s}^{q, t}\left(\partial G, R^{3}\right)$. (Remark that

$$
K_{G}^{\prime} \boldsymbol{\Psi}(\mathbf{x})=\lim _{\epsilon \searrow 0} \int_{\partial G \backslash B(\mathbf{x} ; \epsilon)} K^{G}(\mathbf{y}, \mathbf{x}) \Psi(\mathbf{y}) \mathrm{d} \mathcal{H}_{2}(\mathbf{y})
$$

for $\boldsymbol{\Psi} \in L^{q}\left(\partial G, R^{3}\right)$.) If $\boldsymbol{\Psi} \in L^{q}\left(\partial G, R^{3}\right)$ then

$$
\left[T\left(E_{G} \boldsymbol{\Psi}, Q_{G} \boldsymbol{\Psi}\right)\right]_{ \pm} \mathbf{n}^{G}= \pm \frac{1}{2} \boldsymbol{\Psi}-K_{G}^{\prime} \boldsymbol{\Psi}
$$


(A detailed proof of these relations can be found in [9].)

Let now $\boldsymbol{\Psi} \in H^{-1 / 2}\left(\partial G, R^{3}\right)$. According to [32], Proposition 4.2 we have

$$
\int_{G_{+}}\left[\left(2 \hat{\nabla} E_{G} \boldsymbol{\Psi}\right) \cdot(\hat{\nabla} \mathbf{v})-\left(Q_{G} \boldsymbol{\Psi}\right)(\nabla \cdot \mathbf{v})\right] d \mathcal{H}_{m}=\left\langle\frac{1}{2} \boldsymbol{\Psi}-K_{G}^{\prime} \boldsymbol{\Psi}, \mathbf{v}\right\rangle
$$

for all $\mathbf{v} \in \tilde{W}^{1,2}\left(R^{3}, R^{3}\right)$. Since $\mathcal{C}_{c}^{\infty}\left(R^{3}, R^{3}\right)$ is a dense subset of $\tilde{W}^{1,2}\left(R^{3}, R^{3}\right)$, the behavior of a hydrodynamical single layer potential at infinity gives

$$
\int_{G_{-}}\left[\left(2 \hat{\nabla} E_{G} \boldsymbol{\Psi}\right) \cdot(\hat{\nabla} \mathbf{v})-\left(Q_{G} \boldsymbol{\Psi}\right)(\nabla \cdot \mathbf{v})\right] d \mathcal{H}_{m}=\left\langle\frac{1}{2} \boldsymbol{\Psi}+K_{G}^{\prime} \boldsymbol{\Psi}, \mathbf{v}\right\rangle
$$

for all $\mathbf{v} \in \tilde{W}^{1,2}\left(R^{3}, R^{3}\right)$.

\section{INTEGRAL REPRESENTATION FORMULA}

We shall study a transmission problem also in Besov spaces. We shall need an integral representation formula. Suppose that $0<s<1$, and $1<q, t<\infty$. The trace is a bounded linear operator from $B_{s+1 / p}^{q, t}(G)$ to $B_{s}^{q, t}(\partial G)$ (see [36], Theorem 2.5.2).

If $\mathbf{v}, p$ is an $L^{q}$-solution of the Neumann problem $-\Delta \mathbf{v}+\nabla p=0, \nabla \cdot p=0$ in $G, T(\mathbf{v}, p) \mathbf{n}^{G}=\mathbf{h}$ on $\partial G$ (i.e $\mathbf{v}, p$ is a solution of the homogeneous Stokes system, $\mathbf{v}_{G}^{*}+(\nabla \mathbf{v})_{G}^{*}+p_{\Omega}^{*} \in L^{p}(\partial G)$ and the boundary condition is fulfilled in the sense of the non-tangential limit) then

$$
\left\langle T(\mathbf{v}, p) \mathbf{n}^{G}, \boldsymbol{\Phi}\right\rangle=\int_{\partial G}\left[T(\mathbf{v}, p) \mathbf{n}^{G}\right] \cdot \boldsymbol{\Phi}=2 \int_{G} \hat{\nabla} \boldsymbol{\Phi} \cdot \hat{\nabla} \mathbf{v}-\int_{G} p(\nabla \cdot \boldsymbol{\Phi})
$$

for all $\boldsymbol{\Phi} \in \mathcal{C}_{c}^{\infty}\left(R^{3}, R^{3}\right)$. If $\mathbf{v} \in B_{s+1 / q}^{q, t}\left(G, R^{3}\right), p \in B_{s+1 / q-1}^{q, t}(G),-\Delta \mathbf{v}+\nabla p=0$, $\nabla \cdot \mathbf{v}=0$ in $G$ then the stress $T(\mathbf{v}, p) \mathbf{n}^{G}$ defined by (4.1) is a distribution supported on $\partial G$. Moreover, $(\mathbf{v}, p) \mapsto T(\mathbf{v}, p) \mathbf{n}^{G}$ is a bounded linear operator from $\{[\mathbf{v}, p] \in$ $B_{s+1 / q}^{q, t}\left(G, R^{3}\right) \times B_{s+1 / q-1}^{q, t}(G) ;-\Delta \mathbf{v}+\nabla p=0, \nabla \cdot \mathbf{v}=0$ in $\left.G\right\}$ to $B_{s-1}^{q, t}\left(\partial G, R^{3}\right.$ ) (see [36], Proposition 10.2.1).

Lemma 4.1. Let $-\Delta \mathbf{v}=\nabla p, \nabla \cdot \mathbf{v}=0$ in $R^{3} \backslash F$ where $F$ is compact. If $\mathbf{v}(\mathbf{x}) \rightarrow 0$, $p(\mathbf{x}) \rightarrow 0$ as $|\mathbf{x}| \rightarrow \infty$ or $\mathbf{v} \in \tilde{W}^{1,2}\left(R^{3} \backslash F, R^{3}\right), p \in L^{2}\left(R^{3} \backslash F\right)$, then $|\mathbf{v}(\mathbf{x})|=O\left(|\mathbf{x}|^{-1}\right)$, $|\nabla \mathbf{v}(\mathbf{x})|+|p(\mathbf{x})|=O\left(|\mathbf{x}|^{-2}\right)$ as $|\mathbf{x}| \rightarrow \infty$.

Proof. Fix $r>0$ such that $F \subset B(0 ; r)$. According to [36], Theorem 9.2.3 there exists $\tilde{\mathbf{v}}, \tilde{p}$ such that $-\Delta \tilde{\mathbf{v}}=\nabla \tilde{p}, \nabla \cdot \tilde{\mathbf{v}}=0$ in $R^{3} \backslash \overline{B(0 ; r)}, \tilde{\mathbf{v}}=\mathbf{v}$ on $\partial B(0 ; r)$ and $|\tilde{\mathbf{v}}(\mathbf{x})|=O\left(|\mathbf{x}|^{-1}\right),|\nabla \tilde{\mathbf{v}}(\mathbf{x})|+|\tilde{p}(\mathbf{x})|=O\left(|\mathbf{x}|^{-2}\right)$ as $|\mathbf{x}| \rightarrow \infty$. We have $\tilde{\mathbf{v}}=\mathbf{v}$ and $p-\tilde{p}$ is constant by [28], Theorem 6.5. Thus $p=\tilde{p}$.

Lemma 4.2. Let $0<s<1,1<q, t<\infty$, and $\mathbf{v}_{+} \in B_{s+1 / q}^{q, t}\left(G_{+}, R^{3}\right), p_{+} \in$ $B_{s+1 / q-1}^{q, t}\left(G_{+}\right), \mathbf{v}_{-} \in B_{s+1 / q}^{q, t}\left(G_{-} \cap B(0 ; r), R^{3}\right), p_{-} \in B_{s+1 / q-1}^{q, t}\left(G_{-} \cap B(0 ; r)\right)$ for all 
$r>0$. Suppose that $\mathbf{v}_{ \pm}, p_{ \pm}$is a solution of the transmission problem (1.1), (1.2), (1.9). Then

$$
\begin{gathered}
D_{G_{ \pm}} \mathbf{v}_{ \pm}(\mathbf{x})+E_{G_{ \pm}}\left[T\left(\mathbf{v}_{ \pm}, p_{ \pm}\right)\left( \pm \mathbf{n}^{G}\right)\right](\mathbf{x})= \begin{cases}\mathbf{v}_{ \pm}(\mathbf{x}), & \mathbf{x} \in G_{ \pm} \\
0, & \mathbf{x} \in G_{\mp}\end{cases} \\
\Pi_{G_{ \pm}} \mathbf{v}_{ \pm}(\mathbf{x})+Q_{G_{ \pm}}\left[T\left(\mathbf{v}_{ \pm}, p_{ \pm}\right)\left( \pm \mathbf{n}^{G}\right)\right](\mathbf{x})= \begin{cases}p_{ \pm}(\mathbf{x}), & \mathbf{x} \in G_{ \pm} \\
0, & \mathbf{x} \in G_{\mp}\end{cases} \\
\mathbf{v}_{ \pm}(\mathbf{x})=D_{G} \mathbf{g}(\mathbf{x})+E_{G} \mathbf{\Psi}(\mathbf{x}), \\
\mathbf{x} \in G_{ \pm}, \\
p_{ \pm}(\mathbf{x})=\prod_{G} \mathbf{g}(\mathbf{x})+Q_{G} \mathbf{\Psi}(\mathbf{x}),
\end{gathered}
$$

where

$$
\boldsymbol{\Psi}=T\left(\mathbf{v}_{+}, p_{+}\right) \mathbf{n}-T\left(\mathbf{v}_{-}, p_{-}\right) \mathbf{n} .
$$

Proof. First we deduce the relations (4.2), (4.3) for $\mathbf{v}_{+}, p_{+}$. For $\mathbf{x} \in G_{+}$see [36], Proposition 10.6.1. Suppose now that $\mathbf{x} \in G_{-}$. Using (4.1) for $\mathbf{v}=\mathbf{v}_{+}, p=p_{+}$, $\mathbf{\Phi}(\mathbf{y})=\left(E_{1 k}(\mathbf{y}-\mathbf{x}), E_{2 k}(\mathbf{y}-\mathbf{x}), E_{3 k}(\mathbf{y}-\mathbf{x})\right)^{T}$ and then for $\mathbf{v}(\mathbf{y})=\left(E_{1 k}(\mathbf{y}-\mathbf{x}), E_{2 k}(\mathbf{y}-\right.$ $\left.\mathbf{x}), E_{3 k}(\mathbf{y}-\mathbf{x})\right)^{T}, p(\mathbf{y})=Q_{k}(\mathbf{y}-\mathbf{x}), \mathbf{\Phi}=\mathbf{v}_{+}$and subtracting, we obtain (4.2). Using (4.1) for $\mathbf{v}=\mathbf{v}_{+}, p=p_{+}, \mathbf{\Phi}(\mathbf{y})=\left(Q_{1}(\mathbf{y}-\mathbf{x}), Q_{2}(\mathbf{y}-\mathbf{x}), Q_{3}(\mathbf{y}-\mathbf{x})\right)^{T}$ and then for $\mathbf{v}(\mathbf{y})=\left(Q_{1}(\mathbf{y}-\mathbf{x}), Q_{2}(\mathbf{y}-\mathbf{x}), Q_{3}(\mathbf{y}-\mathbf{x})\right)^{T}, p(\mathbf{y})=0, \boldsymbol{\Phi}=\mathbf{v}_{+}$and subtracting, we obtain (4.3).

We now show relations (4.2), (4.3) for $\mathbf{v}_{-}, p_{-}$. We use (4.2), (4.3) for $G_{-} \cap B(0 ; r)$. Since $\left|\mathbf{v}_{-}(\mathbf{x})\right|=O\left(|\mathbf{x}|^{-1}\right),\left|\nabla \mathbf{v}_{-}(\mathbf{x})\right|+\left|p_{-}(\mathbf{x})\right|=O\left(|\mathbf{x}|^{-2}\right)$ as $|\mathbf{x}| \rightarrow \infty$ by Lemma 4.1, letting $r \rightarrow \infty$ gives (4.2), (4.3).

Adding (4.2), (4.3) we obtain (4.4), (4.5).

Corollary 4.1. Let $0<s<1,1<q, t<\infty, \mathbf{g} \in B_{s}^{q, t}\left(\partial G, R^{3}\right)$. Denote $\mathbf{v}_{ \pm}=D_{G} \mathbf{g}$, $p_{ \pm}=\Pi_{G} \mathbf{g}$ in $G_{ \pm}$. Then $T\left(\mathbf{v}_{+}, p_{+}\right) \mathbf{n}=T\left(\mathbf{v}_{-}, p_{-}\right) \mathbf{n}$ on $\partial G$.

Proof. We have $\mathbf{g}=\left[D_{G} \mathbf{g}\right]_{+}-\left[D_{G} \mathbf{g}\right]_{-}$by (3.6). Put $\boldsymbol{\Psi}=T\left(\mathbf{v}_{+}, p_{+}\right) \mathbf{n}-T\left(\mathbf{v}_{-}, p_{-}\right) \mathbf{n}$. Then $D_{G} \mathbf{g}=D_{G} \mathbf{g}+E_{G} \boldsymbol{\Psi}, \Pi_{G} \mathbf{g}=\Pi_{G} \mathbf{g}+Q_{G} \boldsymbol{\Psi}$ in $R^{3} \backslash \partial G$ by Lemma 4.2. Hence $E_{G} \boldsymbol{\Psi} \equiv 0$, and $Q_{G} \boldsymbol{\Psi}=0$ in $R^{3} \backslash \partial G$. Thus $\boldsymbol{\Psi}=\left[\boldsymbol{\Psi} / 2-K_{G}^{\prime} \boldsymbol{\Psi}\right]-\left[-\boldsymbol{\Psi} / 2-K_{G}^{\prime} \boldsymbol{\Psi}\right]=$ $\left[T\left(E_{G} \boldsymbol{\Psi}, Q_{G} \boldsymbol{\Psi}\right)\right]_{+} \mathbf{n}-\left[T\left(E_{G} \boldsymbol{\Psi}, Q_{G} \boldsymbol{\Psi}\right)\right]_{-} \mathbf{n}=0$.

\section{WEAK SOLUTION IN $\tilde{W}^{1,2}\left(\Omega_{ \pm}\right)$}

Suppose that $\Omega$ has Lipschitz boundary. Let $\mathbf{g} \in H^{1 / 2}\left(\partial \Omega, R^{3}\right)=W^{1 / 2,2}\left(\partial \Omega, R^{3}\right)$, $\mathbf{f} \in H^{-1 / 2}\left(\partial \Omega, R^{3}\right)=W^{-1 / 2,2}\left(\partial \Omega, R^{3}\right)$. If $\boldsymbol{\Psi} \in H^{-1 / 2}\left(\partial \Omega, R^{3}\right)$ then $\mathbf{v}_{ \pm}, p_{ \pm}$given by (4.4), (4.5) is a weak solution of the problem (1.1), (1.2) if and only if

$$
a_{+}\left(\frac{1}{2} \boldsymbol{\Psi}-K_{G}^{\prime} \boldsymbol{\Psi}\right)+a_{-}\left(\frac{1}{2} \boldsymbol{\Psi}+K_{G}^{\prime} \boldsymbol{\Psi}\right)=\mathbf{F},
$$

where

$$
\mathbf{F}=\mathbf{f}+\left(a_{-}-a_{+}\right)\left[T\left(D_{G} \mathbf{g}, \Pi_{G} \mathbf{g}\right)\right]_{+} \mathbf{n} \in H^{-1 / 2}\left(\partial \Omega, R^{3}\right)
$$


(see (3.6), (3.7) and Lemma 4.2).

We show that there exists a unique solution $\mathbf{v}_{ \pm} \in \tilde{W}^{1,2}\left(G_{ \pm}, R^{3}\right), p_{ \pm} \in L^{2}\left(G_{ \pm}\right)$of the problem (1.1), (1.2). If $1 / 2<s<1$ then $\mathbf{v}_{+} \in B_{s}^{2,2}\left(G_{+}, R^{3}\right), p_{+} \in B_{s-1}^{2,2}\left(G_{+}\right)$, $\mathbf{v}_{-} \in B_{s}^{2,2}\left(G_{-} \cap B(0 ; r), R^{3}\right), p_{-} \in B_{s-1}^{2,2}\left(G_{-} \cap B(0 ; r)\right)$ for all $r>0$. Lemma 4.1 gives (1.9). So we have the representation (4.4), (4.5) where $\boldsymbol{\Psi}$ is given by (4.6). For a calculation of the solution we need to solve the equation (5.1). We show that this equation can be solved by the successive approximation method. For this aim we rewrite this equation as

$$
\boldsymbol{\Psi}=\frac{2\left(a_{+}-a_{-}\right)}{\left(a_{+}+a_{-}\right)} K_{G}^{\prime} \boldsymbol{\Psi}+\frac{2}{a_{+}+a_{-}} \mathbf{F} .
$$

We use the following notation: If $X$ is a Banach space denote by $I$ the identity operator on $X$. If $M$ is a subspace of $X$ denote by $\operatorname{dim} M$ the dimension of $M$. If $Y$ is a subspace of $X$ such that $X=M \bigoplus Y$, i.e. $X$ is the direct sum of $M$ and $Y$, denote by $\operatorname{codim} Y=\operatorname{dim} M$ the codimension of $Y$. If $T$ is a bounded linear operator in $X$, denote by $\operatorname{Ker} T=\{x \in X ; T x=0\}$ the kernel of $T, \alpha(T)=\operatorname{dim} \operatorname{Ker} T$, $\beta(T)=\operatorname{codim} T(X)$. We say that $T$ is Fredholm if $T(X)$ is a closed subset of $X$ and $\alpha(T)<\infty, \beta(T)<\infty$. For a Fredholm operator $T$ denote $i(T)=\alpha(T)-\beta(T)$ the index of $T$. If $X$ is a complex Banach space denote by $\sigma(T)$ the spectrum of $T$ and by $r(T)=\sup \{|\lambda| ; \lambda \in \sigma(T)\}$ the spectral radius of $T$.

Proposition 5.1. Suppose that $\mathbf{u}_{ \pm} \in \tilde{W}^{1,2}\left(G_{ \pm}, R^{3}\right), p_{ \pm} \in L^{2}\left(G_{ \pm}, R^{1}\right)$, is a weak solution of the transmission problem for the Stokes system (1.1), (1.2). If $\mathbf{g}=0$, $\mathbf{f}=0$ then $\mathbf{u}_{ \pm}=0, p_{ \pm}=0$.

Proof. Put $\mathbf{v}=\mathbf{u}_{ \pm}$on $G_{ \pm}$. Since $\mathbf{u}_{+}-\mathbf{u}_{-}=0$ on $\partial G$, we have $\mathbf{v} \in \tilde{W}^{1,2}\left(R^{3}, R^{3}\right)$. Since $\nabla \cdot \mathbf{v}=0$, we obtain

$$
0=\langle\mathbf{f}, \mathbf{v}\rangle=a_{+} \int_{G_{+}} 2\left|\hat{\nabla} \mathbf{u}_{+}\right|^{2} d \mathcal{H}_{3}+a_{-} \int_{G_{-}} 2\left|\hat{\nabla} \mathbf{u}_{-}\right|^{2} d \mathcal{H}_{3} .
$$

Thus $\hat{\nabla} \mathbf{v}=0$ on $R^{3} \backslash \partial G$. If $V$ is a component of $R^{3} \backslash \partial G$ then there exists a skew symmetric matrix $A$ (i.e. $A^{T}=-A$ ) and a vector $\mathbf{b}$ such that $\mathbf{v}(\mathbf{x})=A \mathbf{x}+\mathbf{b}$ in $V$ (see for example [32], Lemma 3.1). Suppose first that $V$ is unbounded. Since $\mathbf{v} \in L^{6}\left(V, R^{3}\right)$, we infer that $A=0, \mathbf{b}=0$. Let now $V_{1}, \ldots, V_{k}$ are all components of $R^{3} \backslash \partial \Omega$ on which $\mathbf{v}=0$. Denote by $D$ the closure of $V_{1} \cup \cdots \cup V_{k}$. Suppose that $D \neq R^{3}$. Let $S$ be a component of $\partial D$. Then $\mathbf{v}=0$ on $S$. Choose a component $V$ of $R^{3} \backslash \partial G$ such that $S \subset \partial V$ and $D \cap V=\varnothing$. Suppose that $\mathbf{v}(\mathbf{x})=A \mathbf{x}+\mathbf{b}$ in $V$. Let $W$ be a bounded component of $R^{3} \backslash S$. Put $\mathbf{w}(\mathbf{x})=A \mathbf{x}+\mathbf{b}$ in $W$. Then $\Delta \mathbf{w}=0$ in $W, \mathbf{w}=0$ on $\partial W=S$. The maximum principle for harmonic functions gives that $\mathbf{w}=0$ in $W$. Since $V \subset W$, we have $\mathbf{v}=0$ in $V$, what is a contradiction. Thus $\mathbf{v}=0$ in $R^{3}$.

The Stokes system gives $\nabla p_{ \pm}=\Delta \mathbf{u}_{ \pm}=0$. Thus $p_{ \pm}$is constant on each component of $G_{ \pm}$. Since $p_{-} \in L^{2}\left(G_{-}, R^{1}\right)$, we deduce $p_{-}=0$ on the unbounded component of $G_{-}$. 
Let $S$ be a component of $\partial G$. Then there are a component $V_{+}$of $G_{+}$and a component $V_{-}$of $G_{-}$such that $S=\partial V_{+} \cap \partial V_{-}$. Let $c_{+}, c_{-}$be such constants that $p_{+}=c_{+}$on $V_{+}, p_{-}=c_{-}$on $V_{-}$. Since $\mathbf{u}_{ \pm}, p_{ \pm}$is a classical solution of the transmission problem for the Stokes system (1.1), (1.2), we have $0=a_{+} T\left(\mathbf{u}_{+}, p_{+}\right) \mathbf{n}-a_{-} T\left(\mathbf{u}_{-}, p_{-}\right) \mathbf{n}=$ $\left(a_{-} c_{-}-a_{+} c_{+}\right) \mathbf{n}$ on $S$. Therefore $a_{-} c_{-}=a_{+} c_{+}$. Since $p_{-}=0$ on an unbounded component of $G_{-}$, we deduce that $p_{+}=0, p_{-}=0$.

Lemma 5.1. Let $\boldsymbol{\Psi} \in H^{-1 / 2}\left(\partial G, C^{3}\right)$. Then

$$
\left\langle\boldsymbol{\Psi}, E_{G} \overline{\boldsymbol{\Psi}}\right\rangle=2 \int_{R^{3} \backslash \partial G}\left|\hat{\nabla} E_{G} \boldsymbol{\Psi}\right|^{2} d \mathbf{y} \geq 0 .
$$

If $\left\langle\boldsymbol{\Psi}, E_{G} \overline{\boldsymbol{\Psi}}\right\rangle=0$ then $E_{G} \boldsymbol{\Psi}=0$ in $R^{3}$ and for each component $S$ of $\partial G$ there exists a constant $c_{S}$ such that $\mathbf{\Psi}=c_{s} \mathbf{n}^{G}$ on $S$.

Proof. For (5.4) see [32], Corollary 4.4. Let now $\left\langle\boldsymbol{\Psi}, E_{G} \overline{\boldsymbol{\Psi}}\right\rangle=0$. Then $\hat{\nabla} E_{G} \boldsymbol{\Psi}=0$ in $R^{3} \backslash \partial G$. If $V$ is a component of $R^{3} \backslash \partial G$ then there is a matrix $A$ and a vector b such that $E_{G} \boldsymbol{\Psi}(\mathbf{x})=A \mathbf{x}+\mathbf{b}$ in $V$ (see [32], Lemma 2.1). Denote by $V_{1}, \ldots, V_{k}$ all components of $R^{3} \backslash \partial G$ and suppose that $V_{1}$ is unbounded. Since $E_{G} \mathbf{\Psi}(\mathbf{x}) \rightarrow 0$ as $|\mathbf{x}| \rightarrow \infty$, we infer that $E_{G} \boldsymbol{\Psi}=0$ in $V_{1}$. Denote $D=\cup\left\{V_{j} ; E_{G} \boldsymbol{\Psi} \equiv 0\right.$ in $\left.V_{j}\right\}$. The boundary behavior of a hydrodynamical single layer potential gives $E_{G} \boldsymbol{\Psi}=0$ on cl $D$. Suppose now that cl $D \neq R^{3}$. Fix a component $S$ of $\partial D$. Choose a component $V_{j}$ of $R^{3} \backslash \partial G$ such that $S \subset \partial V_{j}$ and $D \cap V_{j}=\varnothing$. Then there is a matrix $A$ and a vector $\mathbf{b}$ such that $E_{G} \Psi(\mathbf{x})=A \mathbf{x}+\mathbf{b}$ in $V_{j}$. Put $\mathbf{u}(\mathbf{x})=A \mathbf{x}+\mathbf{b}$. Denote by $U$ the component of $R^{3} \backslash S$ such that $V_{j} \subset U$. Then $\mathbf{u}$ is a solution of the problem $\Delta \mathbf{u}=0$ in $U, \mathbf{u}=0$ on $\partial U$. The maximum principle for harmonic functions gives that $\mathbf{u}=0$ in $U$. Thus $E_{G} \boldsymbol{\Psi}=0$ in $V_{j}$, what is a contradiction. So, cl $D=R^{3}$.

Since $\mathbf{u}=E_{G} \boldsymbol{\Psi}, p=Q_{G} \boldsymbol{\Psi}$ is a solution of the Stokes system in $R^{3} \backslash \partial G$, we have $\nabla Q_{G} \boldsymbol{\Psi}=\Delta E_{G} \boldsymbol{\Psi}=0$ in $R^{3} \backslash \partial G$. So, there are constants $c_{j}$ such that $Q_{G} \boldsymbol{\Psi}=c_{j}$ in $V_{j}$. Let now $S$ be a component of $\partial G$. Choose $j$ and $k$ such that $V_{j} \subset G_{+}, V_{k} \subset G_{-}$ and $S=\partial V_{j} \cap V_{k}$. According to boundary behavior of a hydrodynamical potential we have on $S$

$$
\begin{aligned}
\boldsymbol{\Psi} & =\left[\frac{1}{2} \boldsymbol{\Psi}-K_{G}^{\prime} \boldsymbol{\Psi}\right]-\left[-\frac{1}{2} \boldsymbol{\Psi}-K_{G}^{\prime} \boldsymbol{\Psi}\right] \\
& =\left[T\left(E_{G} \boldsymbol{\Psi}, Q_{G} \boldsymbol{\Psi}\right)\right]_{+} \mathbf{n}^{G}-\left[T\left(E_{G} \boldsymbol{\Psi}, Q_{G} \boldsymbol{\Psi}\right)\right]_{-} \mathbf{n}^{G} \\
& =T\left(0, c_{j}\right) \mathbf{n}^{G}-T\left(0, c_{k}\right) \mathbf{n}^{G}=-c_{j} \mathbf{n}^{G}+c_{k} \mathbf{n}^{G} .
\end{aligned}
$$

Lemma 5.2. If $\lambda \in C$ is an eigenvalue of $(1 / 2) I-K_{G}^{\prime}$ in $H^{-1 / 2}\left(\partial G, C^{3}\right)$ then $0 \leq \lambda \leq 1$. 
Proof. Let $\boldsymbol{\Psi}$ be an eigenfunction corresponding to an eigenvalue $\lambda$. According to Lemma 5.1 and [32], Proposition 4.3

$$
\begin{aligned}
2 \int_{G}\left|\hat{\nabla} E_{G} \boldsymbol{\Psi}(x)\right|^{2} d \mathbf{x} & =\left\langle\frac{1}{2} \boldsymbol{\Psi}-K_{G}^{\prime} \boldsymbol{\Psi}, E_{G} \overline{\boldsymbol{\Psi}}\right\rangle \\
& =\left\langle\lambda \boldsymbol{\Psi}, E_{G} \overline{\boldsymbol{\Psi}}\right\rangle=2 \lambda \int_{R^{3} \backslash \partial G}\left|\hat{\nabla} E_{G} \boldsymbol{\Psi}\right|^{2} d \mathbf{x} .
\end{aligned}
$$

If $\left\langle\Psi, E_{G} \bar{\Psi}\right\rangle \neq 0$ then

$$
\lambda=\frac{\int_{G}\left|\hat{\nabla} E_{G} \boldsymbol{\Psi}\right|^{2} d \mathbf{x}}{\int_{R^{3} \backslash \partial G}\left|\hat{\nabla} E_{G} \boldsymbol{\Psi}\right|^{2} d \mathbf{x}}
$$

and $0 \leq \lambda \leq 1$. Let now $\left\langle\boldsymbol{\Psi}, E_{G} \bar{\Psi}\right\rangle=0$. Then for each component $S$ of $\partial G$ there exists a constant $c_{S}$ such that $\boldsymbol{\Psi}=c_{S} \mathbf{n}^{G}$ on $S$. By virtue of (3.3) we obtain that $\lambda=1$ or $\lambda=0$.

Proposition 5.2. Let $X$ be a complex Banach space. Denote by $\mathcal{N}$ the set of all norms on $X$ equivalent to the original norm. If $T$ is a bounded linear operator on $X$ then

$$
r(T)=\inf _{\|\cdot\| \in \mathcal{N}}\|T\|
$$

(See [15].)

Theorem 5.1. Let $\mathbf{f} \in H^{-1 / 2}\left(\partial G, R^{3}\right), \mathbf{g} \in H^{1 / 2}\left(\partial G, R^{3}\right)$. Then there exists a unique weak solution $\mathbf{v}_{ \pm} \in \tilde{W}^{1,2}\left(G_{ \pm}, R^{3}\right), p_{ \pm} \in L^{2}\left(G_{ \pm}, R^{1}\right)$ of the transmission problem for the Stokes system (1.1), (1.2). We have the representation (4.4), (4.5) where $\boldsymbol{\Psi}$ is given by (4.6). Remark that $\boldsymbol{\Psi}$ is a unique solution of the integral equation (5.3) with $\mathbf{F}$ given by (5.2). Fix $\boldsymbol{\Psi}_{0} \in H^{-1 / 2}\left(\partial G, R^{3}\right)$. Set

$$
\boldsymbol{\Psi}_{k}=\frac{2\left(a_{+}-a_{-}\right)}{\left(a_{+}+a_{-}\right)} K_{G}^{\prime} \boldsymbol{\Psi}_{k-1}+\frac{2}{a_{+}+a_{-}} \mathbf{F} .
$$

Then $\boldsymbol{\Psi}_{k} \rightarrow \boldsymbol{\Psi}$ in $H^{-1 / 2}\left(\partial G, R^{3}\right)$. Put

$$
\alpha=\frac{\max \left(a_{+}, a_{-}\right)}{a_{+}+a_{-}} .
$$

Then there exists a constant $C$ dependent only on $G, a_{+}$and $a_{-}$such that

$$
\left\|\Psi_{k}-\Psi\right\|_{H^{-1 / 2}\left(\partial G, R^{3}\right)} \leq C \alpha^{k}\left(\left\|\Psi_{0}\right\|_{H^{-1 / 2}\left(\partial G, R^{3}\right)}+\|F\|_{H^{-1 / 2}\left(\partial G, R^{3}\right)}\right) .
$$

Proof. If $\lambda \in C \backslash\langle 0,1\rangle$ then $(1 / 2) I-K_{G}^{\prime}-\lambda I$ is a Fredholm operator with index 0 by [32], Theorem 4.12 and [37], $\S 16$, Theorem 16. Since $\lambda$ is not an eigenvalue of 
$(1 / 2) I-K_{G}^{\prime}$ by Lemma 5.2 , we deduce that $\lambda \notin \sigma\left((1 / 2) I-K_{G}^{\prime}\right)$. Easy calculation gives

$$
\sigma\left(\frac{2\left(a_{+}-a_{-}\right)}{a_{+}+a_{-}} K_{G}^{\prime}\right) \subset\left\langle-\frac{\left|a_{+}-a_{-}\right|}{a_{+}+a_{-}}, \frac{\left|a_{+}-a_{-}\right|}{a_{+}+a_{-}}\right\rangle .
$$

Proposition 5.2 gives that there exists an equivalent norm \|\| on $H^{-1 / 2}\left(\partial G, C^{3}\right)$ such that

$$
\left\|\frac{2\left(a_{+}-a_{-}\right)}{a_{+}+a_{-}} K_{G}^{\prime}\right\|<\alpha .
$$

Since $\alpha<1$ the equation (5.3) is uniquely solvable (see [16], Theorem 1.3.10). If $\boldsymbol{\Psi}$ is a solution of this equation then $\mathbf{v}, p$ given by (4.4), (4.5) is a solution of the transmission problem (1.1), (1.2). This solution is unique by Proposition 5.1. Lemma 4.2 gives that $\boldsymbol{\Psi}$ is given by (4.6).

Fix a constant $C_{1}$ such that $\|\mathbf{h}\| \leq C_{1}\|\mathbf{h}\|_{H^{-1 / 2}\left(\partial \Omega, R^{3}\right)},\|\mathbf{h}\|_{H^{-1 / 2}\left(\partial \Omega, R^{3}\right)} \leq C_{1}\|\mathbf{h}\|$ for all $\mathbf{h} \in H^{-1 / 2}\left(\partial \Omega, R^{3}\right)$. Denote $C_{2}=2+2 /\left(a_{+}+a_{-}\right)$. According to [16], Theorem 1.3 .10 we have $\boldsymbol{\Psi}_{k} \rightarrow \boldsymbol{\Psi}$ and

$$
\begin{aligned}
& \left\|\boldsymbol{\Psi}-\boldsymbol{\Psi}_{n}\right\|_{H^{-1 / 2}\left(\partial G, R^{3}\right)} \leq C_{1}\left\|\boldsymbol{\Psi}-\boldsymbol{\Psi}_{n}\right\| \leq C_{1} \frac{\alpha^{n}}{1-\alpha}\left\|\boldsymbol{\Psi}_{1}-\boldsymbol{\Psi}_{0}\right\| \\
& \leq \alpha^{n} \frac{C_{1} C_{2}}{1-\alpha}\left[\left\|\boldsymbol{\Psi}_{0}\right\|+\|\mathbf{F}\|\right] \leq \alpha^{n} \frac{C_{1}^{2} C_{2}}{1-\alpha}\left[\left\|\boldsymbol{\Psi}_{0}\right\|_{H^{-1 / 2}\left(\partial G, R^{3}\right)}+\|\mathbf{F}\|_{H^{-1 / 2}\left(\partial G, R^{3}\right)}\right] .
\end{aligned}
$$

\section{The transmission PROBlem in Besov spaCeS}

Lemma 6.1. Let $X, Y$ be Banach spaces, $T$ be a bounded linear Fredholm operator in $X$ with index $0, \mathcal{T}$ be a bounded linear Fredholm operator in $Y$ with index 0 . Let $X \subset Y, Y^{\prime} \subset X^{\prime}$ and $T x=\mathcal{T} x$ for each $x \in X, T^{\prime} z=\mathcal{T}^{\prime} z$ for each $z \in Y^{\prime}$. Then the kernel of $T$ and $\mathcal{T}$ are the same.

(See [31], Lemma 9.)

Theorem 6.1. Suppose that $G$ has boundary of class $\mathrm{C}^{1}, 0<s<1,1<q, t<\infty$. Let $\mathbf{f} \in B_{s-1}^{q, t}\left(\partial G, R^{3}\right), \mathbf{g} \in B_{s}^{q, t}\left(\partial G, R^{3}\right)$. Then there exists a unique solution $\mathbf{v}_{ \pm}$ $p_{ \pm}$of the transmission problem for the Stokes system (1.1), (1.2), (1.9) such that $\mathbf{v}_{+} \in B_{s+1 / q}^{q, t}\left(G_{+}, R^{3}\right), p_{+} \in B_{s+1 / q-1}^{q, t}\left(G_{+}\right), \mathbf{v}_{-} \in B_{s+1 / q}^{q, t}\left(G_{-} \cap B(0 ; r), R^{3}\right), p_{-} \in$ $B_{s+1 / q-1}^{q, t}\left(G_{-} \cap B(0 ; r)\right)$ for all $r>0$. We have the representation (4.4), (4.5) where $\boldsymbol{\Psi}$ is given by (4.6). Remark that $\boldsymbol{\Psi}$ is a unique solution of the integral equation (5.3) in $B_{s-1}^{q, t}\left(\partial G, R^{3}\right)$ with $\mathbf{F}$ given by (5.2). Fix $\mathbf{\Psi}_{0} \in B_{s-1}^{q, t}\left(\partial G, R^{3}\right)$. Let $\boldsymbol{\Psi}_{k}$ be given by (5.5). Then $\boldsymbol{\Psi}_{k} \rightarrow \boldsymbol{\Psi}$ in $B_{s-1}^{q, t}\left(\partial G, R^{3}\right)$. Let $\alpha$ be given by (5.6). Then there exists $a$ constant $C$ dependent only on $G, a_{+}, a_{-}, q, t$ and $s$ such that

$$
\left\|\Psi_{k}-\Psi\right\|_{B_{s-1}^{q, t}\left(\partial G, R^{3}\right)} \leq C \alpha^{k}\left(\left\|\Psi_{0}\right\|_{B_{s-1}^{q, t}\left(\partial G, R^{3}\right)}+\|F\|_{B_{s-1}^{q, t}\left(\partial G, R^{3}\right)}\right) .
$$


Proof. Put $t^{\prime}=t /(t-1), q^{\prime}=q /(q-1)$. Then $K_{G}$ is a compact operator on $B_{1-s}^{q^{\prime}, t^{\prime}}\left(\partial G, C^{3}\right)$ by [29], p. 232. Since $K_{G}^{\prime}$ is the adjoint operator of $K_{G}$, the $K_{G}^{\prime}$ is compact on $B_{s-1}^{q, t}\left(\partial G, C^{3}\right)$. Lemma 6.1 and Lemma 5.2 gives (5.7) in $B_{s-1}^{q, t}\left(\partial G, C^{3}\right)$. So, the equation (5.3) is uniquely solvable in $B_{s-1}^{q, t}\left(\partial G, C^{3}\right)$. If $\mathbf{F}$ is given by (5.2) and $\mathbf{v}_{ \pm}, p_{ \pm}$by (4.4), (4.5), then $\mathbf{v}_{ \pm}, p_{ \pm}$is a solution of the transmission problem for the Stokes system (1.1), (1.2), (1.9) such that $\mathbf{v}_{+} \in B_{s+1 / q}^{q, t}\left(G_{+}, R^{3}\right), p_{+} \in B_{s+1 / q-1}^{q, t}\left(G_{+}\right)$, $\mathbf{v}_{-} \in B_{s+1 / q}^{q, t}\left(G_{-} \cap B(0 ; r), R^{3}\right), p_{-} \in B_{s+1 / q-1}^{q, t}\left(G_{-} \cap B(0 ; r)\right)$ for all $r>0$. Lemma 4.2 gives that $\boldsymbol{\Psi}$ is given by (4.6).

Let now $\mathbf{v}_{ \pm}, p_{ \pm}$be a solution of the transmission problem for the Stokes system (1.1), (1.2), (1.9) with $\mathbf{g}=0, \mathbf{f}=0$. Lemma 4.2 gives (4.4), (4.5) with $\boldsymbol{\Psi}$ given by (4.6). So, (5.3) holds with $\mathbf{F}=0$. Unique solvability of this integral equation forces $\boldsymbol{\Psi}=0$. Hence $\mathbf{v}_{ \pm}=0, p_{ \pm}=0$.

We show (6.1) in the same way as in the proof of Theorem 5.1.

\section{The problem in homogeneous Sobolev Spaces}

Let $\Omega \subset R^{3}$ be an open set, $1<q<\infty$. The space $D^{1, p}(\Omega)$ consists of those functions in $L_{l o c}^{1}(\Omega)$ for which all generalized derivatives of the order 1 are in $L^{q}(\Omega)$. We have $D^{1, q}\left(G_{+}\right)=W^{1, q}\left(G_{+}\right)$but $D^{1, q}\left(G_{-}\right) \neq W^{1, q}\left(G_{-}\right)$(see $\S 1.5 .2, \S 1.5 .3$ and $\S 1.5 .4$ in [30]). We choose $r>0$ such that $\partial G \subset B(0 ; r)$ and define

$$
\|f\|_{D^{1, q}\left(G_{-}\right)}=\left[\int_{G_{-} \cap B(0 ; r)}|f|^{q} \mathrm{~d} \mathcal{H}_{3}+\int_{G_{-}}|\nabla f|^{q} \mathrm{~d} \mathcal{H}_{3}\right]^{1 / q} .
$$

$D^{1, q}\left(G_{-}\right)$equipped with this norm is a Banach space (see [30], §1.5.3). Remark that $\tilde{W}^{1,2}\left(G_{ \pm}\right)$is a subspace of $D^{1,2}\left(G_{ \pm}\right)$and the norm of $\tilde{W}^{1,2}\left(G_{ \pm}\right)$is equivalent with the norm induced from $D^{1,2}\left(G_{ \pm}\right)$(see [30], §1.5.3 and [27], Theorem 8.3).

We shall look for a solution $\mathbf{v}_{ \pm} \in D^{1, q}\left(G_{ \pm}, R^{3}\right), p_{ \pm} \in L^{q}\left(G_{ \pm}\right)$of the transmission problem (1.1), (1.2), (1.9). We shall see that such solution satisfies $\mathbf{v}_{+} \in B_{1}^{q, q}\left(G_{+}, R^{3}\right)$, $p_{+} \in B_{0}^{q, q}\left(G_{+}\right), \mathbf{v}_{-} \in B_{1}^{q, q}\left(G_{-} \cap B(0 ; r), R^{3}\right), p_{-} \in B_{0}^{q, q}\left(G_{-} \cap B(0 ; r)\right)$ for all $r>0$ (see Proposition 7.1). So, Theorem 6.1 gives us represantability of the solution by (4.4), (4.5). Moreover, according to Theorem 6.1 we can solve the integral equation (5.3) by the successive approximation and the estimate (6.1) holds in $W^{-1 / q, q}\left(\partial G, R^{3}\right)$.

Proposition 7.1. Let $1<q<\infty$, $\mathbf{g} \in W^{1-1 / q, q}\left(\partial G, R^{3}\right)=B_{1-1 / q}^{q, q}\left(\partial G, R^{3}\right)$, $\mathbf{f} \in$ $W^{-1 / q, q}\left(\partial G, R^{3}\right)=B_{-1 / q}^{q, q}\left(\partial G, R^{3}\right), \mathbf{v}_{ \pm} \in \mathcal{C}^{\infty}\left(G_{ \pm}, R^{3}\right), p_{ \pm} \in \mathcal{C}^{\infty}\left(G_{ \pm}\right)$. Then the assertion (1) implies the assertion (2). If $3 / 2<q<\infty$ then the assertions (1) and (3) are equivalent. If $q=2$ then the assertions (1), (2) and (3) are equivalent.

1. $\mathbf{v}_{ \pm} \in D^{1, q}\left(G_{ \pm}, R^{3}\right), p_{ \pm} \in L^{q}\left(G_{ \pm}\right)$is a solution of the transmission problem (1.1), (1.2), (1.9). 
2. $\mathbf{v}_{ \pm}, p_{ \pm}$is a solution of the transmission problem (1.1), (1.2), (1.9) such that $\mathbf{v}_{+} \in B_{1}^{q, q}\left(G_{+}, R^{3}\right), p_{+} \in B_{0}^{q, q}\left(G_{+}\right), \mathbf{v}_{-} \in B_{1}^{q, q}\left(G_{-} \cap B(0 ; r), R^{3}\right), p_{-} \in$ $B_{0}^{q, q}\left(G_{-} \cap B(0 ; r)\right)$ for all $r>0$.

3. $\mathbf{v}_{ \pm} \in \tilde{W}^{1,2}\left(G_{ \pm}, R^{3}\right), p_{ \pm} \in L^{2}\left(G_{ \pm}\right)$is a solution of the transmission problem (1.1), (1.2).

Proof. Fix $0<s<1-1 / q$. In all cases $\mathbf{v}_{ \pm}, p_{ \pm}$is a solution of the transmission problem (1.1), (1.2), (1.9) such that $\mathbf{v}_{+} \in B_{s+1 / q}^{q, q}\left(G_{+}, R^{3}\right), p_{+} \in B_{s+1 / q-1}^{q, q}\left(G_{+}\right), \mathbf{v}_{-} \in$ $B_{s+1 / q}^{q, q}\left(G_{-} \cap B(0 ; r), R^{3}\right), p_{-} \in B_{s+1 / q-1}^{q, q}\left(G_{-} \cap B(0 ; r)\right)$ for all $r>0$. (See Lemma 4.1 and the inclusions of Bessel spaces in [45].) According to Lemma 4.2 we have the representation (4.4), (4.5) with $\boldsymbol{\Psi}$ given by (4.6). Since $\mathbf{g} \in W^{1-1 / q, q}\left(\partial G, R^{3}\right)=$ $B_{1-1 / q}^{q, q}\left(\partial G, R^{3}\right), \Psi \in W^{-1 / q, q}\left(\partial G, R^{3}\right)=B_{-1 / q}^{q, q}\left(\partial G, R^{3}\right)$, the properties of hydrodynamical potentials give the assertion.

Theorem 7.1. Let $3 / 2<q<\infty$. If $q \neq 2$ suppose that $G$ has boundary of class $\mathrm{C}^{1}$. Let $\mathbf{g} \in W^{1-1 / q, q}\left(\partial G, R^{3}\right), \mathbf{f} \in W^{-1 / q, q}\left(\partial G, R^{3}\right)$. Then there exists a unique solution $\mathbf{v}_{ \pm} \in D^{1, q}\left(\Omega_{ \pm}\right) p_{ \pm} \in L^{q}\left(\Omega_{ \pm}\right)$of the transmission problem for the Stokes system (1.1), (1.2), (1.9). We have the representation (4.4), (4.5) where $\boldsymbol{\Psi}$ is given by (4.6). Remark that $\boldsymbol{\Psi}$ is a unique solution of the integral equation (5.3) in $W^{-1 / q, q}\left(\partial G, R^{3}\right)$ with $\mathbf{F}$ given by (5.2). Moreover,

$$
\left\|\mathbf{v}_{ \pm}\right\|_{D^{1, p}\left(G_{ \pm}, R^{3}\right)}+\left\|p_{ \pm}\right\|_{L^{p}\left(G_{ \pm}\right)} \leq C\left[\|\mathbf{g}\|_{W^{1-1 / q, q}\left(\partial G, R^{3}\right)}+\|\mathbf{f}\|_{W^{-1 / q, q}\left(\partial G, R^{3}\right)}\right]
$$

where $C$ depends only on $G$ and $q$.

Proof. According to Proposition 7.1, Theorem 5.1 and Theorem 6.1 it is enough to prove (7.1). For $\mathbf{f} \in W^{-1 / q, q}\left(\partial G, R^{3}\right), \mathbf{g} \in W^{1-1 / q, q}\left(\partial G, R^{3}\right)$ denote by $\mathbf{v}_{ \pm}^{\mathbf{g}, \mathbf{f}}, p_{ \pm}^{\mathbf{g}, \mathbf{f}}$ the solution of the transmission problem (1.1), (1.2), (1.9). We have the representation (4.4), (4.5). Thus $U_{ \pm}:[\mathbf{g}, \mathbf{f}] \mapsto\left[\mathbf{v}_{ \pm}^{\mathbf{g}, \mathbf{f}}, p_{ \pm}^{\mathbf{g}, \mathbf{f}}\right]$ is a linear operator from $W^{1-1 / q, q}\left(\partial G, R^{3}\right) \times$ $W^{-1 / q, q}\left(\partial G, R^{3}\right)$ to $D^{1, p}\left(G_{ \pm}, R^{3}\right) \times L^{p}\left(G_{ \pm}\right)$. If $\mathbf{x} \in G_{ \pm}, \mathbf{g}_{n} \rightarrow \mathbf{g}, \mathbf{f}_{n} \rightarrow \mathbf{f}$, then

$$
\left[\mathbf{v}_{ \pm}^{\mathbf{g}_{n}, \mathbf{f}_{n}}(\mathbf{x}), p_{ \pm}^{\left.\mathbf{g}_{n}, \mathbf{f}_{n}\right)}(\mathbf{x})\right] \rightarrow\left[\mathbf{v}_{ \pm}^{\mathbf{g}, \mathbf{f}}(\mathbf{x}), p_{ \pm}^{\mathbf{g}, \mathbf{f}}(\mathbf{x})\right]
$$

So, the operator $U_{ \pm}$is closed. The closed graph theorem gives that it is bounded.

Lemma 7.1. For $1<q<\infty$ denote by $X_{q}$ the set of all $\mathbf{f} \in W^{-1 / q, q}\left(\partial G, R^{3}\right)$ satisfying

$$
\langle\mathbf{f}, \mathbf{c}\rangle=0
$$

for every $\mathbf{c} \in R^{3}$. Then $K_{G}^{\prime}\left(X_{q}\right) \subset X_{q}$.

Proof. Put $\mathbf{g}=\mathbf{f} / 2-K_{G}^{\prime} \mathbf{f}$. Then $\mathbf{u}=E_{G} \mathbf{f}, p=Q_{G} \mathbf{f}$ is a solution of the Neumann problem $\Delta \mathbf{u}=\nabla p$ in $G, \nabla \cdot \mathbf{u}=0$ in $G, T(\mathbf{u}, p) \mathbf{n}^{G}=\mathbf{g}$. Hence $\langle\mathbf{g}, \mathbf{c}\rangle=0$ (see for example [36]). So, $K_{G}^{\prime} \mathbf{f}=\mathbf{f} / 2-\mathbf{g} \in X_{q}$.

Theorem 7.2. Suppose that $G$ has boundary of class $\mathcal{C}^{1}, 1<q \leq 3 / 2$. Let $\mathbf{g} \in$ $W^{1-1 / q, q}\left(\partial G, R^{3}\right), \mathbf{f} \in W^{-1 / q, q}\left(\partial G, R^{3}\right)$. Then there exists a solution $\mathbf{v}_{ \pm} \in D^{1, q}\left(\Omega_{ \pm}\right)$ $p_{ \pm} \in L^{q}\left(\Omega_{ \pm}\right)$of the transmission problem for the Stokes system (1.1), (1.2), (1.9) if 
and only if (7.2) holds true. This solution is unique and (7.1) holds with $C$ dependent only on $G$ and $q$. We have the representation (4.4), (4.5) where $\boldsymbol{\Psi}$ is given by (4.6). Remark that $\boldsymbol{\Psi}$ is a unique solution of the integral equation (5.3) in $W^{-1 / q, q}\left(\partial G, R^{3}\right)$ with $\mathbf{F}$ given by (5.2).

Proof. If $\mathbf{v}_{ \pm} \in D^{1, q}\left(\Omega_{ \pm}\right) p_{ \pm} \in L^{q}\left(\Omega_{ \pm}\right)$is a solution of the transmission problem for the Stokes system (1.1), (1.2), (1.9) then $\mathbf{v}_{+} \in B_{1}^{q, q}\left(G_{+}, R^{3}\right), p_{+} \in B_{0}^{q, q}\left(G_{+}\right)$, $\mathbf{v}_{-} \in B_{1}^{q, q}\left(G_{-} \cap B(0 ; r), R^{3}\right), p_{-} \in B_{0}^{q, q}\left(G_{-} \cap B(0 ; r)\right)$ for all $r>0$ (see Proposition 7.1). Theorem 6.1 gives uniqueness of the problem (1.1), (1.2), (1.9) and the representation (4.4), (4.5).

The operator $S=I-2\left(a_{+}-a_{-}\right) /\left(a_{+}+a_{-}\right) K_{G}^{\prime}$ is an isomorphism on $W^{-1 / q, q}\left(\partial G, R^{3}\right)$ $\left(=B_{-1 / q}^{q, q}\left(\partial G, R^{3}\right)\right)$ by Theorem 6.1 . If $X_{q}$ is the space from Lemma 7.1 then $S\left(X_{q}\right) \subset$ $X_{q}$. So, $S\left(X_{q}\right)=X_{q}$. Let now $\mathbf{f} \in X_{q}$. Since $\left\langle\left[T\left(D_{G} \mathbf{g}, \Pi_{G} \mathbf{g}\right]_{+} \mathbf{n}, \mathbf{c}\right\rangle=0\right.$ for all constant c, we have $F \in X_{q}$. Thus $\boldsymbol{\Psi}=S^{-1} \mathbf{F} \in X_{q}$. If $\mathbf{v}_{ \pm}, p_{ \pm}$is given by (4.4), (4.5), then $\mathbf{v}_{ \pm} \in D^{1, q}\left(\Omega_{ \pm}\right) p_{ \pm} \in L^{q}\left(\Omega_{ \pm}\right)$is a solution of the transmission problem (1.1), (1.2), (1.9).

Let now $\mathbf{f} \notin X_{q}$. If $\mathbf{v}_{ \pm} \in D^{1, q}\left(\Omega_{ \pm}\right), p_{ \pm} \in L^{q}\left(\Omega_{ \pm}\right)$is a solution of the transmission problem (1.1), (1.2), (1.9) then it is given by (4.4), (4.5) where $\boldsymbol{\Psi}=S^{-1} F$ (see Theorem 6.1). Clearly, $\left|\nabla D_{G} \mathbf{g}\right| \in L^{q}\left(G_{-}\right), \Pi_{G} \mathbf{g} \in L^{q}\left(G_{-}\right)$. Since $\left[T\left(D_{G} \mathbf{g}, \Pi_{G} \mathbf{g}\right]_{+} \mathbf{n} \in\right.$ $X_{q}$ and $\mathbf{f} \notin X_{q}$ we have $\mathbf{F} \notin X_{q}$. Hence $\boldsymbol{\Psi} \notin X_{q}$. According to properties of hydrodynamical potentials there exist positive constants $C_{1}, C_{2}$ such that $C_{1}|\mathbf{x}|^{-2} \leq$ $\left|\nabla E_{G} \mathbf{\Psi}(\mathbf{x})\right| \leq C_{2}|\mathbf{x}|^{-2}, C_{1}|\mathbf{x}|^{-2} \leq\left|Q_{G} \boldsymbol{\Psi}(\mathbf{x})\right| \leq C_{2}|\mathbf{x}|^{-2}$ at vicinity of infinity. So, $\mathbf{v}_{-} \notin D^{1, q}\left(\Omega_{-}\right), p_{-} \notin L^{q}\left(\Omega_{-}\right)$.

For $\mathbf{f} \in X_{q}, \mathbf{g} \in W^{1-1 / q, q}\left(\partial G, R^{3}\right)$ denote by $\mathbf{v}_{ \pm}^{\mathbf{g}, \mathbf{f}}, p_{ \pm}^{\mathbf{g}, \mathbf{f}}$ the solution of the transmission problem (1.1), (1.2), (1.9). We have the representation (4.4), (4.5). Thus $U_{ \pm}$: $[\mathbf{g}, \mathbf{f}] \mapsto\left[\mathbf{v}_{ \pm}^{\mathbf{g}, \mathbf{f}}, p_{ \pm}^{\mathbf{g}, \mathbf{f}}\right]$ is a linear operator from $X_{q} \times W^{-1 / q, q}\left(\partial G, R^{3}\right)$ to $D^{1, p}\left(G_{ \pm}, R^{3}\right) \times$ $L^{p}\left(G_{ \pm}\right)$. If $\mathbf{x} \in G_{ \pm}, \mathbf{g}_{n} \rightarrow \mathbf{g}, \mathbf{f}_{n} \rightarrow \mathbf{f}$, then we have

$$
\left[\mathbf{v}_{ \pm}^{\mathbf{g}_{n}, \mathbf{f}_{n}}(\mathbf{x}), p_{ \pm}^{\mathbf{g}(n), \mathbf{f}(n)}(\mathbf{x})\right] \rightarrow\left[\mathbf{v}_{ \pm}^{\mathbf{g}, \mathbf{f}}(\mathbf{x}), p_{ \pm}^{\mathbf{g}, \mathbf{f}}(\mathbf{x})\right]
$$

So, the operator $U_{ \pm}$is closed. The closed graph theorem gives that it is bounded.

\section{8. $L^{q}$-SOLUTION OF THE TRANSMISSION PROBLEM}

Theorem 8.1. Suppose that $G$ has boundary of class $\mathcal{C}^{1}, 1<q<\infty$. Let $\mathbf{f} \in$ $L^{q}\left(\partial G, R^{3}\right), \mathbf{g} \in W^{1, q}\left(\partial G, R^{3}\right)$. Then there exists a unique $L^{q}$-solution $\mathbf{v}_{ \pm} p_{ \pm}$of the transmission problem for the Stokes system (1.1), (1.2), (1.9). We have the representation (4.4), (4.5) where $\boldsymbol{\Psi}$ is given by (4.6). Remark that $\boldsymbol{\Psi}$ is a unique solution of the integral equation (5.3) in $L^{q}\left(\partial G, R^{3}\right)$ with $\mathbf{F}$ given by (5.2). Fix $\boldsymbol{\Psi}_{0} \in L^{q}\left(\partial G, R^{3}\right)$. Let $\boldsymbol{\Psi}_{k}$ be given by (5.5). Then $\boldsymbol{\Psi}_{k} \rightarrow \boldsymbol{\Psi}$ in $L^{q}\left(\partial G, R^{3}\right)$. Let $\alpha$ be given by (5.6). Then there exists a constant $C$ dependent only on $G, a_{+}, a_{-}$and $q$ such that

$$
\left\|\Psi_{k}-\boldsymbol{\Psi}\right\|_{L^{q}\left(\partial G, R^{3}\right)} \leq C \alpha^{k}\left(\left\|\Psi_{0}\right\|_{L^{q}\left(\partial G, R^{3}\right)}+\|F\|_{L^{q}\left(\partial G, R^{3}\right)}\right)
$$


Proof. Fix $1 / q<s<1$. If $\mathbf{v}_{ \pm} p_{ \pm}$is an $L^{q}$-solution of the transmission problem (1.1), (1.2), (1.9) then $\mathbf{v}_{+} \in W^{1, q}\left(G_{+}, R^{3}\right) \subset W^{s, q}\left(G_{+}, R^{3}\right)=B_{s}^{q, q}\left(G_{+}, R^{3}\right), p_{+} \in$ $L^{q}\left(G_{+}\right) \subset B_{s-1}^{q, q}\left(G_{+}\right), \mathbf{v}_{-} \in W^{1, q}\left(G_{+} \cap B(0 ; r), R^{3}\right) \subset B_{s}^{q, q}\left(G_{-} \cap B(0 ; r), R^{3}\right), p_{-} \in$ $L^{q}\left(G_{-} \cap B(0 ; r)\right) \subset B_{s-1}^{q, q}\left(G_{-} \cap B(0 ; r)\right)$ for all $r>0$ (see [33], Lemma 4.1). According to Theorem 6.1 an $L^{q}$-solution of the problem (1.1), (1.2), (1.9) is unique.

Put $q^{\prime}=q /(q-1)$. Then $K_{G}$ is a compact operator on $L^{q^{\prime}}\left(\partial G, C^{3}\right)$ by [29], p. 232 . Since $K_{G}^{\prime}$ is the adjoint operator of $K_{G}$, the operator $K_{G}^{\prime}$ is compact on $L^{q}\left(\partial G, C^{3}\right)$. Lemma 6.1 and Lemma 5.2 gives (5.7) in $L^{q}\left(\partial G, C^{3}\right)$. So, the equation (5.3) is uniquely solvable in $L^{q}\left(\partial G, C^{3}\right)$. If $\mathbf{F}$ is given by (5.2) and $\mathbf{v}_{ \pm}, p_{ \pm}$by (4.4), (4.5), then $\mathbf{v}_{ \pm}, p_{ \pm}$ is an $L^{q}$-solution of the transmission problem (1.1), (1.2), (1.9). Lemma 4.2 gives that $\boldsymbol{\Psi}$ is given by (4.6).

We show (8.1) in the same way as in the proof of Theorem 5.1.

Acknowledgment: Supported by GA ČR Grant P201/11/1304 and RVO: 67985840.

\section{REFERENCES}

[1] R. A. Adams and J. L. Fournier, Sobolev Spaces, 2. ed., Elsevier, Oxford, 2003.

[2] D. B. Bogy, Two edge-bounded wedges of different materials and wedge angles under surface tractions, Trans. ASME J. Appl. Mech. 38 (1971), 377-386.

[3] T. K. Chang and D. H. Pahk, Spectral properties for layer potentials associated to the Stokes equation in Lipschitz domains, Manuscripta Math. 130 (2009), 359-373.

[4] O. Chkadua, S. E. Mikhailov and D. Natroshvili, Localized direct segregated boundary-domain integral equations for variable coefficient transmission problems with interface crack, Mem. Differential Equations Math. Phys. 52 (2011), 17-64.

[5] M. Costabel and E. P. Stephan, A direct boundary integral equation method for transmission problems, J. Math. Anal. Appl. 106 (1985), 376-413.

[6] R. Dautray and J. L. Lions, Mathematical Analysis and Numerical Methods for Sciences and Technology, vol. 4, Integral Equations and Numerical Methods, Springer-Verlag, Berlin Heidelberg, 1990.

[7] J. P. Dempsey and G. B. Sinclair, On the singular behavior of the vertex of a bimaterial wedge, J. Elast. 11 (1981), 317-327.

[8] J. Deny, Les potentiels d'énergie finie, Acta Math. 82 (1950), 107-183.

[9] P. Deuring, W. von Wahl and P. Weidemaier, Das lineare Stokes-System im $\mathrm{R}^{3}$ (1. Vorlesungen über das Innenraumproblem), Bayreuther Mathematische Schriften 27 (1988), 1-252.

[10] P. E. Druet, Global Lipschitz continuity for elliptic transmission problems with a boundary intersecting interface, Math. Bohem. 138 (2013), 185-224.

[11] L. Escauriaza, E. Fabes and G. Verchota, On a regularity theorem for weak solutions to transmission problems with internal Lipschitz boundaries, Proc. Amer. Math. Soc. 115 (1992), 1069-1076.

[12] L. Escauriaza and M. Mitrea, Transmission problems and spectral theory for singular integral operators on Lipschitz domains, J. Funct. Anal. 216 (2004), 141-171.

[13] L. Escauriaza and J. K. Seo, Regularity properties of solutions to transmission problems, Trans. Amer. Math. Soc. 338 (1993), 405-430.

[14] G. N. Gatica, A. Márquez and S. Meddahi, Analysis of the coupling of BEM, FEM and mixedFEM for a two-dimensional fluid-solid interaction problem, Appl. Num. Math. 59 (2009), 27352750 .

[15] I. Gohberg and A. Marcus, Some remarks on topologically equivalent norms, Izvestija Mold. Fil. Akad. Nauk SSSR 10 (1960), 91-95. (in Russian) 
[16] W. Hackbush, Integral Equations. Theory and Numerical Treatment, Bikhäuser, Bassel, 1995.

[17] G. C. Hsiao and W. L. Wendland, Boundary Integral Equations, Springer, Heidelberg, 2008.

[18] R. B. Kellogg, On the Poisson equation with intersecting interfaces, Appl. Anal. 4 (1975), $102-129$.

[19] C. E. Kenig, Recent progress on boundary value problems on Lipschitz domains. Pseudodifferential operators and Applications, Proc. Symp., Notre Dame/ Indiana 1984. Proc. Symp. Pure Math. 43 (1985), 175-205.

[20] M. Kohr, M. Lanza de Cristoforis and W. L. Wendland, Nonlinear Neumann-transmission problems for Stokes and Brinkman equations on Euclidean Lipschitz domains, Potential Anal. 38 (2013), 1123-1171.

[21] M. Kohr, C. Pintea and W. L. Wendland, Brinkman-type operators on Riemannian manifolds: Transmission problems in Lipschitz and $C^{1}$ domains, Potential Anal. 32 (2010), 229-273.

[22] M. Kohr, C. Pintea and W. L. Wendland, Stokes-Brinkman transmission problems on Lipschitz and $C^{1}$ domains in Riemannian manifolds, Commun. Pure Appl. Anal. 9 (2010), 493-537.

[23] M. Kohr, C. Pintea and W. L. Wendland, Dirichlet-transmission problems for general Brinkman operators in Lipschitz and $C^{1}$ domains in Riemannian manifolds, Discrete Contin. Dyn. Syst., ser. B, 15 (2011), 999-1018.

[24] M. Kohr, C. Pintea, and W. L. Wendland, Dirichlet-transmission problems for pseudodifferential Brinkman operators on Sobolev and Besov spaces associated to Lipschitz domains in Riemannian manifolds, Z. Angew. Math. Mech. 93 (2013), No. 6-7, 446-458.

[25] M. Kohr, G. P. Raja Sekhar, E. M. Ului, and W. L. Wendland, Two-dimensional StokesBrinkmann cell model - a boundary integral formulation, Appl. Anal. 91 (2012), 251-275.

[26] H. Kozono and H. Sohr, On a new class of generalized solutions for the Stokes equations in exterior domains, Ann. Sc. Norm. Super. Pisa, Cl. Sci. 4 ser. 19 (1998) 155-181.

[27] E. H. Lieb and M. Loss, Analysis, AMS, USA, 1997.

[28] P. Maremonti, R. Russo and G. Starita, On the Stokes equations: the boundary value problem, in: P. Maremonti (Eds.), Advances in Fluid Dynamics, Dipartimento di Matematica Seconda Università di Napoli, 1999, pp. 69-140.

[29] V. Maz'ya, M. Mitrea and T. Shaposhnikova, The inhomogenous Dirichlet problem for the Stokes system in Lipschitz domains with unit normal close to VMO*, Funct. Anal. Appl. 43 (2009), $217-235$.

[30] V. G. Maz'ya and S. V. Poborchi, Differentiable Functions on Bad Domains, World Scientific, Singapore, 1997.

[31] D. Medková, Regularity of solutions of the Neumann problem for the Laplace equation, Le Matematiche, LXI (2006), 287-300.

[32] D. Medková, Convergence of the Neumann series in BEM for the Neumann problem of the Stokes system, Acta Appl. Math. 116 (2011), 281-304.

[33] D. Mitrea, A generalization of Dahlberg's theorem concerning the regularity of harmonic Green potentials, Trans. Amer. Math. Soc. 360 (2008), 3771-3793.

[34] D. Mitrea, M. Mitrea and S. Qiang, Variable coefficient transmission problem and singular integral operators on non-smooth manifolds, J. Integral. Equ. Appl. 18 (2006), 361-397.

[35] I. Mitrea and K. Ott, Counterexamples to the well-posedness of $L^{p}$ transmission boundary value problems for the Laplacian, Proceedings of the Amer. Math. Soc. 135 (2007), 2037-2043.

[36] M. Mitrea and M. Wright, Boundary value problems for the Stokes system in arbitrary Lipschitz domains, Astérisque 344, Paris, 2012.

[37] V. Müller, Spectral Theorey of Linear Operators and Spectral Systems in Banach Algebras, Birkhäuser, Basel, 2007.

[38] D. Munz and Y. Y. Yang, Stress singularities at the interface in bounded dissimilar materials under mechanical and thermal loading, J. Appl. Mech. 59 (1992), 857-861. 
[39] S. Nicaise and A. M. Sändig, General interface problems I/II, Math. Methods Appl. Sci. 17 (1994), 395-450.

[40] S. Nicaise and A. M. Sändig, Transmission problems for the Laplace and elasticity operators: regularity and boundary integral formulation, Math. Models Meth. Appl. Sci. 9 (1999), 855-898.

[41] J. Peetre, New Thougts on Besov Spaces, Duke University Mathematics Series, Durham, 1976.

[42] C. Pozrikidis, Boundary integral and singularity methods for linearized viscous flow, Cambridge texts in Applied Mathematics, Cambridge University Press, New York, 1992.

[43] O. Steinbach, Numerical Approximation Methods for Elliptic Boundary Value Problems. Finite and Boundary Elements, Springer, New York, 2008.

[44] O. Steinbach and W. L. Wendland, On C. Neumann's method for second-order elliptic systems in domains with non-smooth boundaries, J. Math. Anal. Appl. 262 (2001), 733-748.

[45] H. Triebel, Theory of function spaces III, Monographs in Mathematics 100, Basel, Birkhäuser, 2006.

${ }^{1}$ Mathematical Institute,

Academy of Sciences of the Czech Republic,

ŽITNÁ 25,

11567 PRAHA 1 ,

Czech Republic

E-mail address: medkova@math.cas.cz 